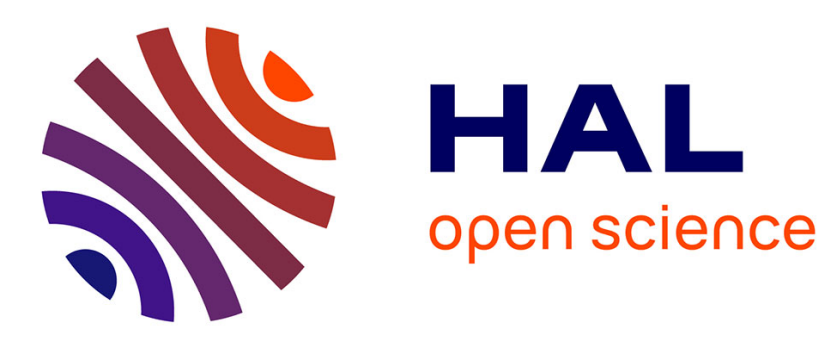

\title{
Analytical Solution for Waves in Planets with Atmospheric Superrotation. I. Acoustic and Inertia-Gravity Waves
}

J. Peralta, T. Imamura, P. L. Read, D. Luz, Arianna Piccialli, M. A. López-Valverde

\section{To cite this version:}

J. Peralta, T. Imamura, P. L. Read, D. Luz, Arianna Piccialli, et al.. Analytical Solution for Waves in Planets with Atmospheric Superrotation. I. Acoustic and Inertia-Gravity Waves. The Astrophysical Journal Supplement, 2014, 213 (1), pp.17. 10.1088/0067-0049/213/1/17 . hal-01023605

\section{HAL Id: hal-01023605 https://hal.science/hal-01023605}

Submitted on 9 Jul 2020

HAL is a multi-disciplinary open access archive for the deposit and dissemination of scientific research documents, whether they are published or not. The documents may come from teaching and research institutions in France or abroad, or from public or private research centers.
L'archive ouverte pluridisciplinaire $\mathbf{H A L}$, est destinée au dépôt et à la diffusion de documents scientifiques de niveau recherche, publiés ou non, émanant des établissements d'enseignement et de recherche français ou étrangers, des laboratoires publics ou privés. 


\title{
ANALYTICAL SOLUTION FOR WAVES IN PLANETS WITH ATMOSPHERIC SUPERROTATION. I. ACOUSTIC AND INERTIA-GRAVITY WAVES
}

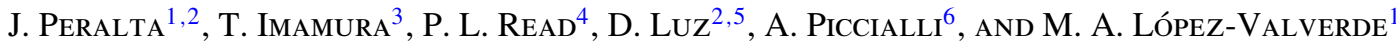 \\ ${ }^{1}$ Instituto de Astrofísica de Andalucía (CSIC), Glorieta de la Astronomía, 18008 Granada, Spain; peralta@ iaa.es \\ ${ }^{2}$ Centro de Astronomia e Astrofísica da Universidade de Lisboa (CAAUL), Observatório Astronómico de Lisboa, Tapada da Ajuda, 1349-018 Lisboa, Portugal \\ ${ }^{3}$ Institute of Space and Astronautical Science-Japan Aerospace Exploration Agency 3-1-1, Yoshinodai, Chuo-ku, Sagamihara, Kanagawa 252-5210, Japan \\ ${ }^{4}$ Department of Physics, University of Oxford, Clarendon Laboratory, Parks Road, Oxford, UK \\ ${ }^{5}$ Faculdade de Ciěncias, Universidade de Lisboa, 1749-016 Lisboa, Portugal \\ ${ }^{6}$ LATMOS, UVSQ, 11 bd dAlembert, 78280 Guyancourt, France \\ Received 2013 December 16; accepted 2014 June 5; published 2014 July 3
}

\begin{abstract}
This paper is the first of a two-part study devoted to developing tools for a systematic classification of the wide variety of atmospheric waves expected on slowly rotating planets with atmospheric superrotation. Starting with the primitive equations for a cyclostrophic regime, we have deduced the analytical solution for the possible waves, simultaneously including the effect of the metric terms for the centrifugal force and the meridional shear of the background wind. In those cases when the conditions for the method of the multiple scales in height are met, these wave solutions are also valid when vertical shear of the background wind is present. A total of six types of waves have been found and their properties were characterized in terms of the corresponding dispersion relations and wave structures. In this first part, only waves that are direct solutions of the generic dispersion relation are studied-acoustic and inertia-gravity waves. Concerning inertia-gravity waves, we found that in the cases of short horizontal wavelengths, null background wind, or propagation in the equatorial region, only pure gravity waves are possible, while for the limit of large horizontal wavelengths and/or null static stability, the waves are inertial. The correspondence between classical atmospheric approximations and wave filtering has been examined too, and we carried out a classification of the mesoscale waves found in the clouds of Venus at different vertical levels of its atmosphere. Finally, the classification of waves in exoplanets is discussed and we provide a list of possible candidates with cyclostrophic regimes.
\end{abstract}

Key words: methods: analytical - methods: data analysis - planets and satellites: atmospheres - waves

Online-only material: color figures

\section{INTRODUCTION}

Slowly rotating planets with atmospheres seem to have in common an intriguing phenomenon called superrotation, consisting of an excess of atmospheric angular momentum at the equator, compared with the angular momentum air would have in the tropics in solid-body rotation with the underlying surface (Read 2011). In our solar system, the most prominent example of atmospheric superrotation is Venus which exhibits a zonal retrograde superrotation with wind speeds up to 60 times faster than the rotation of a solid planet (Schubert 1983). The other body in the solar system known to possess superrotation is Titan, a fact confirmed with in situ measurements (Bird et al. 2005) and ground-based observations (Luz et al. 2006). In slowly rotating bodies like Venus and Titan, the Coriolis terms become small enough to be neglected in the momentum equations (Schubert 1983; Flasar et al. 2010), and the dynamical regime thus becomes dominated by a cyclostrophic balance (i.e., the pressure gradient force is balanced by the metric terms representing the centrifugal force due to strong winds), while in the case of fast rotating planets like the Earth, Mars, and the giant planets of the solar system, the geostrophic balance dominates (pressure gradients are balanced by the Coriolis force). Strong evidence of atmospheric superrotation has also been found in modeling studies of exoplanets (Faigler et al. 2013), both on local and global scales for terrestrial (Joshi et al. 1997; Merlis \& Schneider 2010) and gas giant exoplanets (Showman et al. 2009; Heng et al. 2011; Rauscher \& Menou 2012). Experimental evidence of atmospheric superrotation has also become available recently through Doppler measurements with Spitzer observations (Knutson et al. 2007, 2009).

On top of this superrotation, Venus and Titan also exhibit several types of wavelike motions within and above the clouds (Seiff et al. 1992; Lorenz et al. 2014). In the specific case of Venus, the variety of waves and periodicities is impressive, and they have been detected with observations at different wavelengths through their effects on lower clouds' opacity to thermal radiation and on the upper cloud patterns for reflected sunlight at visible and ultraviolet wavelengths (Belton et al. 1976; Rossow et al. 1980; Del Genio \& Rossow 1990; Peralta et al. 2008; Piccialli et al. 2014); waves have also been detected in winds (Rossow et al. 1990), thermal emission (Taylor et al. 1980; Apt \& Leung 1982), and temperature profiles inferred by probe and balloon motions (Seiff et al. 1992) as well as radio occultation data (Hinson \& Jenkins 1995; Tellmann et al. 2012). Identically to what happens on Earth, atmospheric waves are expected to play a crucial role in the atmospheric circulation and must play a key role in explaining the atmospheric superrotation (Hou \& Farrell 1987), either by accelerating the atmosphere in the case of some global-scale waves, or dragging it via the absorption of waves (Gierasch et al. 1997). To evaluate the waves for the Venus case, a number of analytical and numerical works have been undertaken, studying a wide spectrum of waves in some cases (Imamura 2006) or focusing on specific types of waves such as the low-latitude Y-shaped feature present in UV images (Schubert 1983). Different strategies were followed in these studies: a simple approach in terms of basic dispersion relations classically used for the Earth 
(Del Genio \& Rossow 1990; Peralta et al. 2008; Tellmann et al. 2012), coordinates fixed to the faster Venus winds in order to get back a non-negligible Coriolis factor and, thus, a system of equations resembling the terrestrial case (Covey \& Schubert 1981), equations fitting a cyclostrophic balance (Covey \& Schubert 1982; Smith et al. 1992, 1993), and the detailed study of waves generated by complex general circulation models (GCMs) for Venus (Yamamoto \& Takahashi 2003; Lee et al. 2007; Lebonnois et al. 2010).

Despite the evident interest in the cyclostrophic regime in the field of atmospheric dynamics, the analytical solutions for the expected waves still remain to be fully explored. Unlike the terrestrial case, where the background wind can be assumed to be null as a first approximation, the cyclostrophic balance reveals the superrotating zonal wind to be a key parameter for wave solutions. Most efforts have been devoted to the case of Venus, and even though recent Venus GCMs employ realistic winds, the majority of strictly analytical works for atmospheric waves make use of realistic vertical wind profiles but employ a solid-body rotation approximation for the latitudinal wind profile (Covey \& Schubert 1982; Schubert \& Walterscheid 1984; Schinder et al. 1990; Smith et al. 1993), which is not consistent since real winds on Venus show significant latitudinal, solar local time, and long temporal variations (Hueso et al. 2012; Peralta et al. 2012; Luz et al. 2011; Machado et al. 2012). Indeed, the horizontal shear of the wind is usually included in the GCMs but its specific effect on the waves - combined or not with the centrifugal force-has never been explored in depth. Concerning the dispersion relations for the different types of waves expected in an atmospheric regime in cyclostrophic equilibrium, so far, only the relation for acoustic-gravity waves has been obtained, in a work restricted to small-scale waves and where neither centrifugal forces nor meridional shear of the background wind were considered (Schubert \& Walterscheid 1984). More effort has been devoted to gravity waves than to acoustic waves, mainly because the latter are harder to detect through remote sensing and because they are not expected to be significant in the net budget of energy and momentum in the general circulation. However, by comparing observations and modeling of the acoustic waves and their expected propagation, it is possible to infer environmental information such as the atmospheric structure, composition, or even background dynamics (Petculescu \& Lueptow 2007).

This article is the first of a two-part work in which we present a detailed study of atmospheric waves on a planet with cyclostrophic balance, and even though we focus mainly on Venus, its results can be extended to Titan and exoplanets with a cyclostrophic regime. In this first part, we demonstrate that, under reasonable assumptions and applying the method of the multiple scales in height (Boyd 1978), the dispersion relation for acoustic and inertia-gravity waves can be obtained from a cyclostrophic regime that includes solely the meridional shear of the background zonal flow. The equations for the wave perturbations are deduced in Section 2, and their solutions, together with a generic dispersion relation, are obtained in Section 3. The solutions for acoustic and inertia-gravity waves and their dispersion relations are studied in detail in Section 4, as well as their behavior in limiting cases. We explore some of the classical atmospheric approximations and discuss their validity for wave filtering in Section 5. Finally, we classify some of the mesoscale waves found in the atmosphere of Venus in Section 6, while the main conclusions are presented in Section 7.

\section{WAVE EQUATIONS FOR A CYCLOSTROPHIC ATMOSPHERE}

Assuming that the atmosphere can be described as an ideal gas, atmospheric motions are adiabatic, friction is negligible, and, after applying a proper scale analysis, the system of equations for a cyclostrophic atmosphere are the following (see Appendix A for the deduction in the case of Venus):

$$
\begin{aligned}
\frac{\partial u}{\partial t}+u \frac{\partial u}{\partial x}+v \frac{\partial u}{\partial y}+w \frac{\partial u}{\partial z} & =-\frac{1}{\rho} \frac{\partial P}{\partial x}+\frac{u v}{a} \tan \phi, \\
\frac{\partial v}{\partial t}+u \frac{\partial v}{\partial x}+v \frac{\partial v}{\partial y}+w \frac{\partial v}{\partial z} & =-\frac{1}{\rho} \frac{\partial P}{\partial y}-\frac{u^{2}}{a} \tan \phi, \\
\frac{\partial w}{\partial t}+u \frac{\partial w}{\partial x}+v \frac{\partial w}{\partial y}+w \frac{\partial w}{\partial z} & =-\frac{1}{\rho} \frac{\partial P}{\partial z}-g, \\
\frac{\partial \rho}{\partial t}+u \frac{\partial \rho}{\partial x}+v \frac{\partial \rho}{\partial y}+w \frac{\partial \rho}{\partial z} & =-\rho\left(\frac{\partial u}{\partial x}+\frac{\partial v}{\partial y}+\frac{\partial w}{\partial z}\right), \\
\frac{\partial \Theta}{\partial t}+u \frac{\partial \Theta}{\partial x}+v \frac{\partial \Theta}{\partial y}+w \frac{\partial \Theta}{\partial z} & =0, \\
P & =\rho R T,
\end{aligned}
$$

where Equation (1f) is the equation for ideal gases, and we have expanded the material derivatives in the momentum equations (Equations (1a)-(c)), continuity (Equation (1d)), and thermodynamic equations (Equation (1e)). Here, $(u, v, w)$ are the zonal, meridional, and vertical components of the wind; $P$ is the atmospheric pressure; $\rho$ is the density; $g$ is the gravity acceleration; $\phi$ is the latitude; $z$ is the altitude over the planet's surface; $a$ is the planet's radius; and $\Theta \equiv \ln \theta$, with $\theta$ being the potential temperature. Note that in the case of Titan, Coriolis terms are not as small as in Venus, and in some regions of its atmosphere, they cannot be neglected anymore (Flasar et al. 2010), leading to a gradient flow regime (Holton et al. 2002) instead of a cyclostrophic regime. This more generic situation involves a higher complexity and will be tackled in the future.

To study waves, we follow the standard procedure of applying the method of perturbations, with the disturbances being small enough to neglect cross terms. Furthermore, for simplicity, we will assume that the waves produce perturbations only in the $X-Z$ plane, so we will have $\partial / \partial y=0$ for all the disturbances. In this case, the disturbances for each atmospheric parameter will be

$$
\begin{aligned}
u & =u_{0}\left(y, z_{0}\right)+u^{\prime}(x, z, t) \\
v & =v^{\prime}(x, z, t) \\
w & =w^{\prime}(x, z, t) \\
\rho & =\rho_{0}(z)+\rho^{\prime}(x, z, t) \\
P & =P_{0}(y, z)+P^{\prime}(x, z, t) \\
\Theta & =\Theta_{0}(z)+\Theta^{\prime}(x, z, t),
\end{aligned}
$$

where we are considering the atmosphere locally at a certain height $z_{0}$, and where the atmosphere is at rest except for a zonal background wind that varies only with latitude, i.e., $u_{0}=u_{0}\left(y, z_{0}\right), v_{0}=w_{0}=0$. Unfortunately, keeping the term $\partial u_{0} / \partial z$ in the equations considerably complicates the deduction and precludes a strictly analytical solution for the waves in a cyclostrophic regime - the main aim of this work-when no further atmospheric assumptions are made. Except for those 

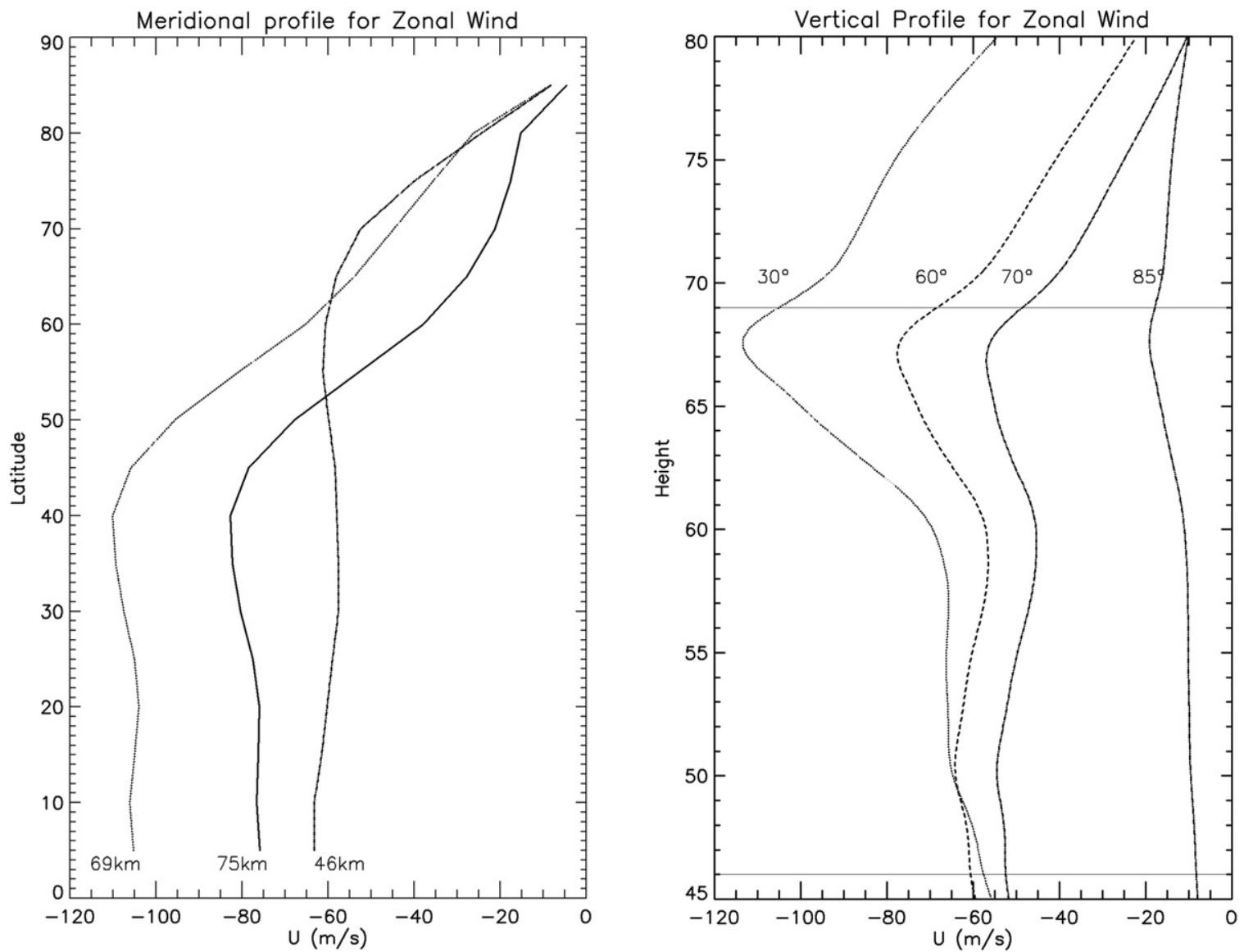

Figure 1. Meridional (left) and vertical profiles (right) for the zonal wind according to our reference atmosphere of Venus (see Appendix A). In the left panel, it can be seen that the strong meridional shear occurs poleward of $45^{\circ}$. In the right panel, we see that the vertical shear is important for lower latitudes, and the zonal wind peaks at about $68 \mathrm{~km}$. The zonal wind has been interpolated using the vertical profiles obtained by the Pioneer Venus probes and data obtained from cloud tracking at the upper and lower clouds (levels marked with horizontal gray lines in the right panel).

regions where $\partial u_{0} / \partial z$ is small enough to be neglected, further assumptions are thus required as shown below. Note that in the specific case of Venus (see Figure 1), the vertical shear can be neglected only at high latitudes and close to the cloud tops at about 67-68 km in height (where the vertical shear changes its sign).

Let us consider now the more general case of non-negligible vertical shear for the background wind, with two situations being possible. The first situation occurs when waves are fast enough not to be distorted by the vertical wind shear, which occurs when the wave's intrinsic phase velocity is higher than the zonal wind change in one vertical wavelength, i.e., $\left|\bar{c}_{k}\right|>\lambda_{z} \cdot\left|\partial u_{0} / \partial z\right|$ (Iga $\&$ Matsuda 2005); when this happens, we can still omit the term $\partial u_{0} / \partial z$ in the meridional momentum equation (see Figure 2 to check whether this condition is met in the cloud region of Venus).

The second situation is when waves become distorted by the vertical wind shear, forcing the usage of the vertical shear of the background zonal wind. When this happens, we must check for the applicability of the so-called method of multiple scales in height (Boyd 1978). For those atmospheric regions where this method is valid, we can proceed with dividing our problem into two steps: first we obtain the wave solution at a given height $z_{0}$ for the problem of only meridional shear of the wind, and second, we reconstruct the vertical structure of waves with a modified version of the WKB method (in this case, with the usual slowly varying WKB amplitude factor modified to account for the effect of the vertical shear of the wind). Because

\section{Limit for wave distortion by vertical shear of zonal wind}

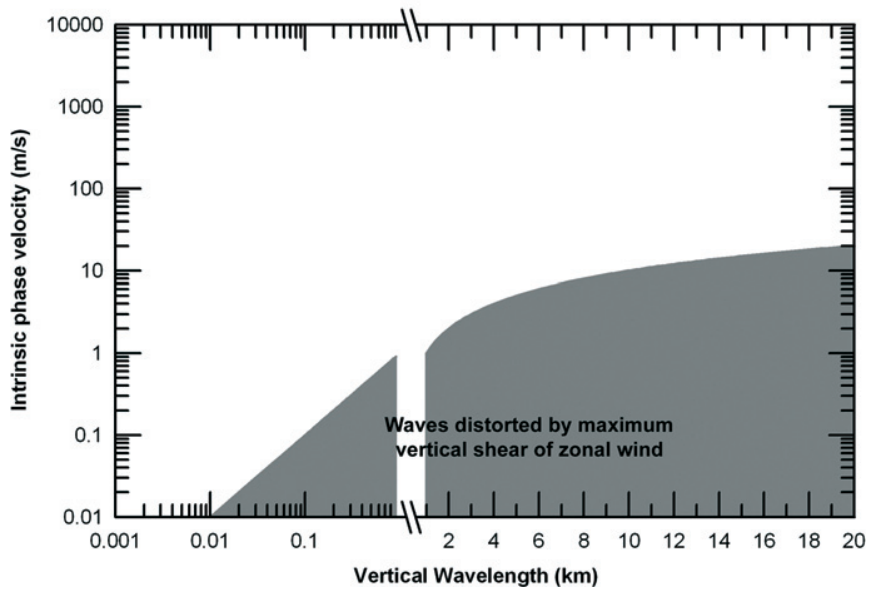

Figure 2. Wave distortion in terms of the intrinsic phase velocity and vertical wavelength of the waves. The white and gray regions indicate, respectively, where waves are unaffected and distorted by the vertical shear of the background zonal wind. The boundary between both regions is defined by the condition $\left|\bar{c}_{k}\right|>\lambda_{z} \cdot\left|\partial u_{0} / \partial z\right|$ (Iga \& Matsuda 2005), where we have used the maximum value of the vertical shear given by our reference atmosphere.

of the extension of this work, we have restricted our study to the first step, i.e., we obtain the wave solution for solely meridional shear, which is sufficient to calculate all the wave properties at a given height except for the overall amplitude and phase factors (Boyd 1978). A summary of the applicability of the method 
of the multiple scales in Venus and its limitations is shown in Appendix B.

Introducing perturbations (Equation (2)) in Equations ((1a)(1f)), neglecting all terms containing products of perturbations, and defining a centrifugal frequency as $\Psi \equiv\left(u_{0} / a\right) \tan \phi$,

$$
\begin{gathered}
\frac{\partial u^{\prime}}{\partial t}+u_{0} \frac{\partial u^{\prime}}{\partial x}+\frac{\partial}{\partial x}\left(\frac{P^{\prime}}{\rho_{0}}\right)-\Psi \cdot v^{\prime}+\frac{\partial u_{0}}{\partial y} v^{\prime}=0, \\
\frac{\partial v^{\prime}}{\partial t}+u_{0} \frac{\partial v^{\prime}}{\partial x}+2 \Psi \cdot u^{\prime}=0, \\
\frac{\partial w^{\prime}}{\partial t}+u_{0} \frac{\partial w^{\prime}}{\partial x}+\frac{1}{\rho_{0}} \frac{\partial P^{\prime}}{\partial z}+\frac{\rho^{\prime}}{\rho_{0}} g=0, \\
\frac{\partial \rho^{\prime}}{\partial t}+u_{0} \frac{\partial \rho^{\prime}}{\partial x}+w^{\prime} \frac{\partial \rho_{0}}{\partial z}=-\rho_{0}\left(\frac{\partial u^{\prime}}{\partial x}+\frac{\partial w^{\prime}}{\partial z}\right), \\
\frac{\partial \Theta^{\prime}}{\partial t}+u_{0} \frac{\partial \Theta^{\prime}}{\partial x}+w^{\prime} \frac{\partial \Theta_{0}}{\partial z}=0, \\
\frac{P^{\prime}}{P_{0}}=\frac{\rho^{\prime}}{\rho_{0}}+\frac{T^{\prime}}{T_{0}},
\end{gathered}
$$

where one of the terms in the meridional momentum equation (Equation (3b)) has been cancelled out by the gravitational force by rotating the coordinate system around the $X$-axis so that the $Z$-axis lies along the vector sum of the gravitational acceleration and the centrifugal force (see Appendix C).

Now, we define the density scale height as $1 / H_{0} \equiv$ $-\partial \ln \rho_{0} / \partial z$, and the atmospheric static stability as $B \equiv$ $\partial \ln \theta / \partial z=\partial \Theta / \partial z$, with $N=\sqrt{g \cdot B}$ being the Brunt-Väisälä frequency (Holton 2004). Equations (3a)-(3f) yield

$$
\begin{gathered}
\frac{\partial u^{\prime}}{\partial t}+u_{0} \frac{\partial u^{\prime}}{\partial x}+\frac{\partial}{\partial x}\left(\frac{P^{\prime}}{\rho_{0}}\right)-\Psi \cdot v^{\prime}+\frac{\partial u_{0}}{\partial y} v^{\prime}=0 \\
\frac{\partial v^{\prime}}{\partial t}+u_{0} \frac{\partial v^{\prime}}{\partial x}+2 \Psi \cdot u^{\prime}=0 \\
n_{4} \cdot\left(\frac{\partial w^{\prime}}{\partial t}+u_{0} \frac{\partial w^{\prime}}{\partial x}\right)+\frac{\partial}{\partial z}\left(\frac{P^{\prime}}{\rho_{0}}\right)-n_{3} \cdot B \frac{P^{\prime}}{\rho_{0}}-g \cdot \Theta^{\prime}=0 \\
n_{2} \cdot\left[\frac{\partial}{\partial t}\left(\frac{\rho^{\prime}}{\rho_{0}}\right)+u_{0} \frac{\partial}{\partial x}\left(\frac{\rho^{\prime}}{\rho_{0}}\right)\right]+\frac{\partial u^{\prime}}{\partial x}+\frac{\partial w^{\prime}}{\partial z}-n_{1} \cdot \frac{w^{\prime}}{H_{0}}=0 \\
\frac{\partial \Theta^{\prime}}{\partial t}+u_{0} \frac{\partial \Theta^{\prime}}{\partial x}+B \cdot w^{\prime}=0
\end{gathered}
$$

and the procedure for obtaining Equation (4c) is explained in detail in Appendix D. Following a method suggested by J. S. A. Green (1970, unpublished lecture notes, Imperial College) and repeatedly used in the bibliography (Green 1999; Norbury \& Roulstone 2002), we marked with the parameters $\left(n_{1}, n_{2}, n_{3}, n_{4}\right)$ those terms that are key to applying important approximations for the atmosphere: $n_{1}$ and $n_{2}$ (together with $n_{3}$, as will be seen later) account for the terms related to an incompressible atmosphere $(D \rho / D t=0)$ and $n_{4}$ is related to the hydrostatic approximation $(D w / D t=0)$. These parameters are meaningless and just act as "tracers" of the terms, being "dragged" along the mathematical procedure until they end up incorporated in the final dispersion relation, thus helping one to understand how each approximation affects the filtering of waves (see Section 5). Their value will be one by default, and combinations of them can be set to zero when we want to apply a specific approximation, as will be shown later.

Finally, we assume that the disturbances due to waves have a periodic behavior, with the form $u^{\prime}(x, z, t)=\hat{u}(z)$. $\exp \left[i \cdot\left(k_{x} x-\omega t\right)\right]$, where the periodic behavior in the vertical direction is included in $\hat{u}(z)$. We study only single modes; $\omega$ is assumed to be real ("neutral" waves); and we regard the atmosphere as unbounded in $x, y$, and $z$. Introducing these disturbances and defining the intrinsic frequency as $\bar{\omega} \equiv$ $\omega-k_{x} u_{0}$, we obtain a set of equations for the wave amplitudes of the disturbances: $\hat{u}(z), \hat{v}(z), \hat{w}(z), \hat{P}(z), \hat{\rho}(z)$, and $\hat{\Theta}(z)$ :

$$
\begin{gathered}
-i \bar{\omega} \cdot \hat{u}+\left(\frac{\partial u_{0}}{\partial y}-\Psi\right) \cdot \hat{v}+i k_{x} \cdot \frac{\hat{P}}{\rho_{0}}=0, \\
-i \bar{\omega} \cdot \hat{v}+2 \Psi \cdot \hat{u}=0, \\
-n_{4} \cdot i \bar{\omega} \cdot \hat{w}+\frac{d}{d z}\left(\frac{\hat{P}}{\rho_{0}}\right)-n_{3} \cdot B \frac{\hat{P}}{\rho_{0}}-g \cdot \hat{\Theta}=0, \\
-n_{2} \cdot i \bar{\omega} \cdot \frac{\hat{\rho}}{\rho_{0}}+i k_{x} \cdot \hat{u}+\frac{d \hat{w}}{d z}-n_{1} \cdot \frac{\hat{w}}{H_{0}}=0, \\
-i \bar{\omega} \cdot \hat{\Theta}+B \cdot \hat{w}=0 .
\end{gathered}
$$

\section{THE GENERAL DISPERSION RELATION FOR WAVES}

In this section, we derive from Equations (5a)-(5e) a general expression for the dispersion relation by following these steps: (1) combining this set of equations to obtain a single differential equation for the wave amplitude of the vertical velocity, $\hat{w}(z)$, (2) solving this equation and obtaining the dispersion relation $\bar{\omega}\left(k_{x}, m\right)$, and (3) the solution $\hat{w}(z)$ can be used in the remaining equations to obtain a solution for the other dependent variables.

First, we solve Equations (5a) and (5b) for the zonal and meridional velocity disturbances in terms of the pressure perturbations. In order to simplify these equations, we define a squared frequency $\xi^{2}=2 \Psi\left(\Psi-\partial u_{0} / \partial y\right)$, i.e., $\xi$ accounts for the centrifugal frequency when it is modified by the meridional shear of the background zonal wind. Also note that the condition $\Psi\left(\Psi-\partial u_{0} / \partial y\right)>0$ is always true; if the meridional shear of the wind is negligible $\left(\partial u_{0} / \partial y \cong 0\right)$, then $\xi^{2} \cong 2 \Psi^{2}$; and, for the equatorial region of Venus, we have $\xi^{2} \cong 0$. Substituting this squared frequency yields

$$
\begin{gathered}
\hat{u}=\left(\frac{\bar{\omega} \cdot k_{x}}{\bar{\omega}^{2}-\xi^{2}}\right) \cdot \frac{\hat{P}}{\rho_{0}}, \\
\hat{v}=-i \cdot\left(\frac{2 \Psi \cdot k_{x}}{\bar{\omega}^{2}-\xi^{2}}\right) \cdot \frac{\hat{P}}{\rho_{0}} .
\end{gathered}
$$

Second, we use the thermodynamic equation (Equation (5e)) to write the vertical momentum equation (Equation $(5 c)$ ) in terms of only $\hat{w}$ and $\hat{P} / \rho_{0}$, thus obtaining the following vertical momentum and continuity equations:

$$
\begin{aligned}
-n_{4} & \cdot i \bar{\omega} \cdot \hat{w}+\frac{d}{d z}\left(\frac{\hat{P}}{\rho_{0}}\right)-n_{3} \cdot B \frac{\hat{P}}{\rho_{0}}+i \frac{g B}{\bar{\omega}} \hat{w}=0, \\
& -n_{2} \cdot i \bar{\omega} \cdot \frac{\hat{\rho}}{\rho_{0}}+i k_{x} \cdot \hat{u}+\frac{d \hat{w}}{d z}-n_{1} \cdot \frac{\hat{w}}{H_{0}}=0 .
\end{aligned}
$$


It can be demonstrated (Holton 2004) that the amplitude for the potential temperature can be expressed as $\hat{\Theta} \equiv \gamma^{-1} \hat{P} / P_{0}-$ $\hat{\rho} / \rho_{0}=c_{S}^{-2} \hat{P} / \rho_{0}-\hat{\rho} / \rho_{0}$ (where $c_{S}^{2} \equiv \gamma R T_{0}$ is the speed of sound, and $\gamma=C_{P} / C_{V}$ ). Thus, using Equation (5e), we obtain $\hat{\rho} / \rho_{0}=c_{S}^{-2} \hat{P} / \rho_{0}+i \cdot(B / \bar{\omega}) \cdot \hat{w}$, which allows one to write the vertical momentum and continuity equations in terms of $\hat{w}$ and $\hat{P} / \rho_{0}$ alone. Moreover, multiplying Equation (7a) by $i \bar{\omega}$, replacing $\hat{u}$ by its expression given in Equation (6a), and sorting the different terms, the vertical momentum and continuity equations can be written in the form

$$
\begin{gathered}
i \bar{\omega} \frac{d}{d z}\left(\frac{\hat{P}}{\rho_{0}}\right)-i \bar{\omega} \cdot n_{3} B \frac{\hat{P}}{\rho_{0}}+\left(n_{4} \bar{\omega}^{2}-g B\right) \cdot \hat{w}=0, \\
\frac{d \hat{w}}{d z}+\left(n_{2} B-\frac{n_{1}}{H_{0}}\right) \cdot \hat{w}-i \bar{\omega}\left(\frac{n_{2}}{c_{S}^{2}}-\frac{k_{x}^{2}}{\bar{\omega}^{2}-\xi^{2}}\right) \cdot \frac{\hat{P}}{\rho_{0}}=0 .
\end{gathered}
$$

Defining a variable $\Gamma$ which does not depend on $z$ and contains some of the terms in Equation (8b),

$$
\Gamma=\frac{n_{2}}{c_{S}^{2}}-\frac{k_{x}^{2}}{\bar{\omega}^{2}-\xi^{2}},
$$

we obtain the following simpler expression for $i \bar{\omega}\left(\hat{P} / \rho_{0}\right)$ and $i \bar{\omega} \cdot d / d z\left(\hat{P} / \rho_{0}\right)$ :

$$
i \bar{\omega} \cdot \frac{\hat{P}}{\rho_{0}}=\frac{1}{\Gamma} \frac{d \hat{w}}{d z}+\frac{1}{\Gamma}\left(n_{2} B-\frac{n_{1}}{H_{0}}\right) \cdot \hat{w}
$$

$i \bar{\omega} \cdot \frac{d}{d z}\left(\frac{\hat{P}}{\rho_{0}}\right)=\frac{1}{\Gamma} \frac{d^{2} \hat{w}}{d z^{2}}+\frac{1}{\Gamma}\left(n_{2} B-\frac{n_{1}}{H_{0}}\right) \cdot \frac{d \hat{w}}{d z}+\frac{n_{2}}{\Gamma} \frac{d B}{d z} \cdot \hat{w}$,

and, substituting these into Equation (8a), we obtain a single equation in terms of $\hat{w}(z)$ :

$$
\begin{aligned}
& \frac{d^{2} \hat{w}}{d z^{2}}+\left[B\left(n_{2}-n_{3}\right)-\frac{n_{1}}{H_{0}}\right] \cdot \frac{d \hat{w}}{d z}+\left[n_{3} B\left(\frac{n_{1}}{H_{0}}-n_{2} B\right)\right. \\
& \left.+n_{2} \frac{d B}{d z}+\Gamma \cdot\left(n_{4} \bar{\omega}^{2}-g B\right)\right] \cdot \hat{w}=0
\end{aligned}
$$

In order to obtain a simpler differential equation without first derivatives, we define $\hat{w}(z)$ as $\hat{w}(z)=\tilde{w}(z)$. $\exp \left\{-(1 / 2) \cdot\left[B\left(n_{2}-n_{3}\right)-n_{1} / H_{0}\right] \cdot z\right\}$. Applying this change of variables, we obtain the following second-order equation:

$$
\frac{d^{2} \tilde{w}}{d z^{2}}+\left\{\begin{array}{l}
n_{3} B \cdot\left(\frac{n_{1}}{H_{0}}-n_{2} B\right)+\left(\frac{n_{2}+n_{3}}{2}\right) \frac{d B}{d z}+\ldots \\
+\Gamma \cdot\left(n_{4} \bar{\omega}^{2}-g B\right)-\frac{1}{4}\left[B\left(n_{2}-n_{3}\right)-\frac{n_{1}}{H_{0}}\right]^{2}
\end{array}\right\} \cdot \tilde{w}=0 .
$$

The second-order equation (Equation (12)) has two different solutions depending on whether we consider that the static stability varies with the altitude or not. If we assume that $B(z)$ varies linearly with height and the generic case of all tracer parameters being set to $1\left(n_{1}=n_{2}=n_{3}=n_{4}=1\right)$, then Equation (12) adopts the form $d^{2} \tilde{w} / d z^{2}-(C \cdot z+D) \cdot \tilde{w}=0$ which, by means of a simple change of variables (Zaitsev \& Polyanin 2002), can be transformed into an Airy equation whose solution will be $\tilde{w}=C_{1} \cdot A i(\zeta)+C_{2} \cdot B i(\zeta)$ where $A i(\zeta)$ and $B i(\zeta)$ are the Airy functions of the first and second kind, respectively.
For simplicity, hereafter, we assume that within the vertical range covered by the atmospheric waves studied in this article (acoustic and inertia-gravity waves) the static stability can be taken as approximately constant with height. In this second case, the solution for Equation (12) is a wave with the form $\tilde{w}(z) \propto \exp (i \cdot m \cdot z)$, whenever the constant multiplying $\tilde{w}$ is real and positive. For this wave solution, we have the following generic dispersion relation:

$$
\begin{aligned}
m^{2}= & n_{3} B \cdot\left(\frac{n_{1}}{H_{0}}-n_{2} B\right)+\left(n_{4} \bar{\omega}^{2}-g B\right) \cdot\left(\frac{n_{2}}{c_{S}^{2}}-\frac{k_{x}^{2}}{\bar{\omega}^{2}-\xi^{2}}\right) \\
& -\frac{1}{4}\left[B\left(n_{2}-n_{3}\right)-\frac{n_{1}}{H_{0}}\right]^{2} .
\end{aligned}
$$

The solution for the vertical wind disturbance is then

$$
w^{\prime} \propto \exp \left\{-\frac{1}{2}\left[B\left(n_{2}-n_{3}\right)-\frac{n_{1}}{H_{0}}\right] \cdot z\right\} \cdot e^{i\left(k_{x} \cdot x+m \cdot z-\omega \cdot t\right)} .
$$

\section{ACOUSTIC, GRAVITY, AND INERTIAL WAVES}

In this section, we study the wave solutions that are derived from the generic expression for the dispersion relation of the atmospheric waves, requiring no additional approximations. Setting all the tracer parameters to $1\left(n_{1}=n_{2}=n_{3}=n_{4}=1\right)$ in the dispersion relation (Equation (13)), we obtain

$$
m^{2}=\frac{k_{x}^{2} \cdot\left(g B-\bar{\omega}^{2}\right)}{\bar{\omega}^{2}-\xi^{2}}+\frac{\bar{\omega}^{2}}{c_{S}^{2}}-\frac{1}{4 H_{0}^{2}},
$$

where we took into account that $B+g / c_{S}^{2}=1 / H_{0}$ (Vallis 2006). The dispersion relation (Equation (15)) is a more general form of the dispersion relation previously obtained by Schubert \& Walterscheid (1984; see their Equation (9)) for acoustic-gravity waves in Venus. The reason is that Equation (15) additionally accounts for the centrifugal force combined with the meridional shear of the background wind through the term $\xi^{2}$. Moreover, there are clear similarities with the general dispersion relation for the terrestrial case, where we have a null background zonal wind $\left(u_{0}=0\right)$ and geostrophic balance (Eckart 1960), where the squared frequency $\xi^{2}$ clearly plays a role similar to the Coriolis factor on the Earth:

$$
m^{2}=\frac{k_{x}^{2} \cdot\left(g B-\omega^{2}\right)}{\omega^{2}-f^{2}}+\frac{\omega^{2}}{c_{S}^{2}}-\frac{1}{4 H_{0}^{2}} .
$$

Solving for $\bar{\omega}$ in Equation (15), we obtain the following biquadratic equation:

$$
\begin{aligned}
\bar{\omega}^{4} & -\bar{\omega}^{2} \cdot\left[\xi^{2}+c_{S}^{2}\left(k_{x}^{2}+m^{2}+\frac{1}{4 H_{0}^{2}}\right)\right] \\
+ & c_{S}^{2}\left[g B \cdot k_{x}^{2}+\xi^{2} \cdot\left(m^{2}+\frac{1}{4 H_{0}^{2}}\right)\right]=0 .
\end{aligned}
$$

Equation (17) can be written in the form of an ordinary quadratic equation by means of a simple change of variables $\bar{\omega}^{2}=\chi$, then admitting two exact solutions for $\bar{\omega}^{2}$ :

$$
\begin{aligned}
\bar{\omega}^{2}= & \frac{1}{2}\left[\xi^{2}+c_{S}^{2}\left(k_{x}^{2}+m^{2}+\frac{1}{4 H_{0}^{2}}\right)\right] \\
& \times\left\{1 \mp \sqrt{1-\frac{4 c_{S}^{2}\left[k_{x}^{2} g B+\xi^{2} \cdot\left(m^{2}+\left(2 H_{0}\right)^{-2}\right)\right]}{\left[\xi^{2}+c_{S}^{2}\left(k_{x}^{2}+m^{2}+\left(2 H_{0}\right)^{-2}\right)\right]^{2}}}\right\} .
\end{aligned}
$$


Table 1

Orders of Magnitude for the Terms Appearing in the Generic Dispersion Relation for Venus

\begin{tabular}{lc}
\hline \hline Terms in Dispersion Relation & Source \\
\hline$\Psi \approx 10^{-5} \leftrightarrow 10^{-6} \mathrm{~s}^{-1} \rightarrow \Psi^{2} \approx 10^{-10} \leftrightarrow 10^{-12} \mathrm{~s}^{-2}$ & Peralta et al. (2007) \\
$u_{0} \approx 10^{2} \leftrightarrow 10^{0} \mathrm{~m} \mathrm{~s}^{-1}$ & Peralta et al. (2007) \\
$\frac{\partial u_{0}}{\partial y} \approx 10^{-5} \leftrightarrow 10^{-6} \mathrm{~s}^{-1}$ & Peralta et al. (2007) \\
$H_{0} \approx 6380 \mathrm{~m} \rightarrow \frac{1}{4 H_{0}^{2}} \approx 10^{-9} \mathrm{~m}^{-2}$ & Seiff et al. (1985) \\
$c_{S} \approx 252 \mathrm{~m} \mathrm{~s}^{-1} \rightarrow c_{S}^{2} \approx 6.4 \times 10^{4} \mathrm{~m}^{2} \mathrm{~s}^{-2}$ & Seiff et al. (1985) \\
$B \approx 10^{-6} \mathrm{~m}^{-1} \leftrightarrow 10^{-5} \mathrm{~m}^{-1}$ & Piccialli (2010) \\
$g \approx 8.87 \mathrm{~m} \mathrm{~s}^{-2}$ & Seiff et al. (1985) \\
$k_{x}^{2} \geqslant 10^{-14} \mathrm{~m}^{-2}$ & \\
$m^{2} \geqslant 10^{-9} \mathrm{~m}^{-2}$ & \\
$\xi^{2} \approx 10^{-10} \mathrm{~s}^{-2}$ & \\
\hline
\end{tabular}

Notes. They have been evaluated using data from the Venus International Reference Atmosphere (VIRA), radio-occultation data from Venus Express, and wind speeds measured with cloud tracking in remote sensing images.

At this stage of the demonstration, expression (18) can be further simplified depending on the planet studied. If we apply a scale analysis suitable for the atmosphere of Venus (see Table 1), then the terms in the fraction under the square root of Equation (18) can be simplified as $c_{S}^{2} \cdot \xi^{2} \cdot\left(m^{2}+\left(2 \mathrm{H}_{0}\right)^{-2}\right) \sim$ $10^{-13}$ and $c_{S}^{2} \cdot g B \cdot k_{x}^{2} \sim 10^{-13}$, thus the numerator is $\sim 10^{-13}$. On the other hand, we also have that $c_{S}^{2} \cdot\left(k_{x}^{2}+m^{2}+\left(2 H_{0}\right)^{-2}\right) \sim$ $10^{-4}$, so the denominator is $\sim 10^{-8}$.

As a result, the square root term has the form $\sqrt{1-X}$ with $X \ll 1$, which allows using a Taylor expansion around $X=0$ :

$$
\sqrt{1-X}=1-\frac{X}{2}+\mathrm{O}\left(X^{2}\right) \approx 1-\frac{X}{2} .
$$

The following solutions are obtained, one $\left(\bar{\omega}_{g}\right)$ corresponding to the inertia-gravity waves, and another $\left(\bar{\omega}_{a}\right)$ to the acoustic waves:

$$
\begin{aligned}
& \bar{\omega}_{g}^{2} \approx \frac{g B \cdot k_{x}^{2}+\xi^{2} \cdot\left[m^{2}+\left(2 H_{0}\right)^{-2}\right]}{k_{x}^{2}+m^{2}+\left(2 H_{0}\right)^{-2}}, \\
& \bar{\omega}_{a}^{2} \approx\left[k_{x}^{2}+m^{2}+\left(2 H_{0}\right)^{-2}\right] \cdot c_{S}^{2} .
\end{aligned}
$$

The dispersion relation (Equation (20a)) provides a kind of wave that depends on both the static stability $(B)$ and the background zonal wind via $\xi$ that is a function of the meridional shear of the zonal wind $\left(d u_{0} / d y\right)$ and the centrifugal force $(\Psi)$. As the restoring forces are, in this case, the buoyancy and the inertial forces, these waves are classified as inertia-gravity waves. They are displayed for different vertical wavenumbers $(m)$ in the dispersion graphs shown in Figure 3, which allows one to determine the ranges of intrinsic phase velocity and horizontal wavelengths for which these waves are possible in the case of Venus. As the dispersion relation (Equation (20a)) also depends on the magnitude of the background wind, its horizontal shear, and the static stability, the dispersion graph will also vary with latitude and altitude. It can be seen that the inertia-gravity waves, as for the terrestrial case, are clearly limited by the adiabatic speed of sound $\left(c_{S}\right)$, the Brunt-Väisälä frequency $(\sqrt{g B})$, and the centrifugal frequency modified by the horizontal shear of the zonal wind $(\xi)$. The area shadowed light gray corresponds to the values where the assumption $\bar{\omega}^{2} \gg \xi^{2}$ is no longer valid.

It is also of interest to study some limiting cases for the inertia-gravity waves and how they affect the final form of the dispersion relation (see Table 2), sometimes being pure gravity waves and in other cases inertial waves, as observed in the geostrophic case (Holton 2004). Indeed, pure inertial waves are more apparent in the case of large horizontal wavelengths, which can be influenced by the meridional shear of the zonal wind and by the centrifugal force (inertial), while pure gravity waves are more common for waves of smaller spatial scale. We also highlight that inertial waves cannot exist at the equator or when the atmosphere is at rest, while gravity waves are only possible when $B>0$.

Similarly, as is done for the inertial waves in a geostrophic atmosphere (Holton 2004), the necessary conditions for the existence of inertial waves on a cyclostrophic one can be studied from the point of view of an inertial stability, depending on the value of

$$
\Psi \cdot\left(\Psi-\frac{\partial u_{0}}{\partial y}\right) \equiv \begin{cases}>0 & \text { stable } \\ =0 & \text { neutral } \\ <0 & \text { unstable }\end{cases}
$$

Note that this term is quite similar to the one defined in the geostrophic case (Holton 2004) and defined as $f \cdot\left(f-\partial u_{g} / \partial y\right)$ with $u_{g}$ being the geostrophic zonal wind and $f$ the Coriolis factor. In fact, if we consider the generic form of Ertel's potential vorticity $q=\rho_{0}^{-1} \cdot[2 \vec{\Omega}+\vec{\nabla} \times \vec{V}] \cdot \vec{\nabla} \theta_{0}$ (Holton et al. 2002) expressed in spherical coordinates (Gill 1982), it can be directly demonstrated that for a slowly rotating planet with atmospheric superrotation in the zonal direction, Ertel's potential vorticity reduces to the expression $q=\rho_{0}^{-1} \cdot\left[\Psi-\left(\partial u_{0} / \partial y\right)\right] \cdot \partial \theta_{0} / \partial z$ and Equation (21) turns out to be part of the general expression for the potential vorticity.

The dispersion relation (Equation (20b)) describes waves that do not depend on the value of static stability but on the adiabatic speed of sound $\left(c_{S}\right)$, i.e., these are acoustic waves for which the restoring force is the atmospheric pressure. They can be displayed for different vertical wavenumbers $(m)$ in the dispersion graph exhibited in Figure 3. Note that due to the terms $H_{0}$ and the adiabatic speed of sound $\left(c_{S}\right)$, the dispersion relation (Equation (20b)) will also depend on the atmospheric region to be studied, and the dispersion graph for acoustic waves will slightly change with latitude and, especially, with altitude. The acoustic waves are limited by the adiabatic speed of sound $\left(c_{S}\right)$ and by the Brunt-Väisälä frequency. As was done during the analysis of the inertia-gravity waves, we can also study limiting cases for the acoustic waves (see Table 3 ). For long wavelengths (i.e., $K=\sqrt{k_{x}^{2}+m^{2}}$ is very small, with $K$ being the total wavenumber) the acoustic waves are dispersive and the group velocity is zero (thus they are unable to transport energy or momentum by themselves); moreover, it is straightforward to demonstrate that the propagation is almost vertical for acoustic waves with large horizontal wavelengths. On the other hand, acoustic waves with small wavelengths (i.e., very large $K$ ) are described by the classical dispersion relation for acoustic waves. Their intrinsic phase velocity is equal to the adiabatic speed of sound (i.e., these waves are pressure perturbations that propagate at the speed of sound relative to the background zonal wind), they are non-dispersive, in the horizontal plane their phase velocity and group velocity are the same, they are longitudinal waves (air parcels are disturbed perpendicularly to the wave fronts), and their propagation is horizontal $\left(\cos \alpha_{Z}=k_{x} / \sqrt{k_{x}^{2}+m^{2}} \cong 1\right)$.

\subsection{Limitations from the Meridional Variation of $\xi^{2}$}

The meridional shear of the background zonal wind has been demonstrated to affect the wave solution for a cyclostrophic 
Dispersion Graph for Waves at $05.0^{\circ}$ and $65.0 \mathrm{~km}$
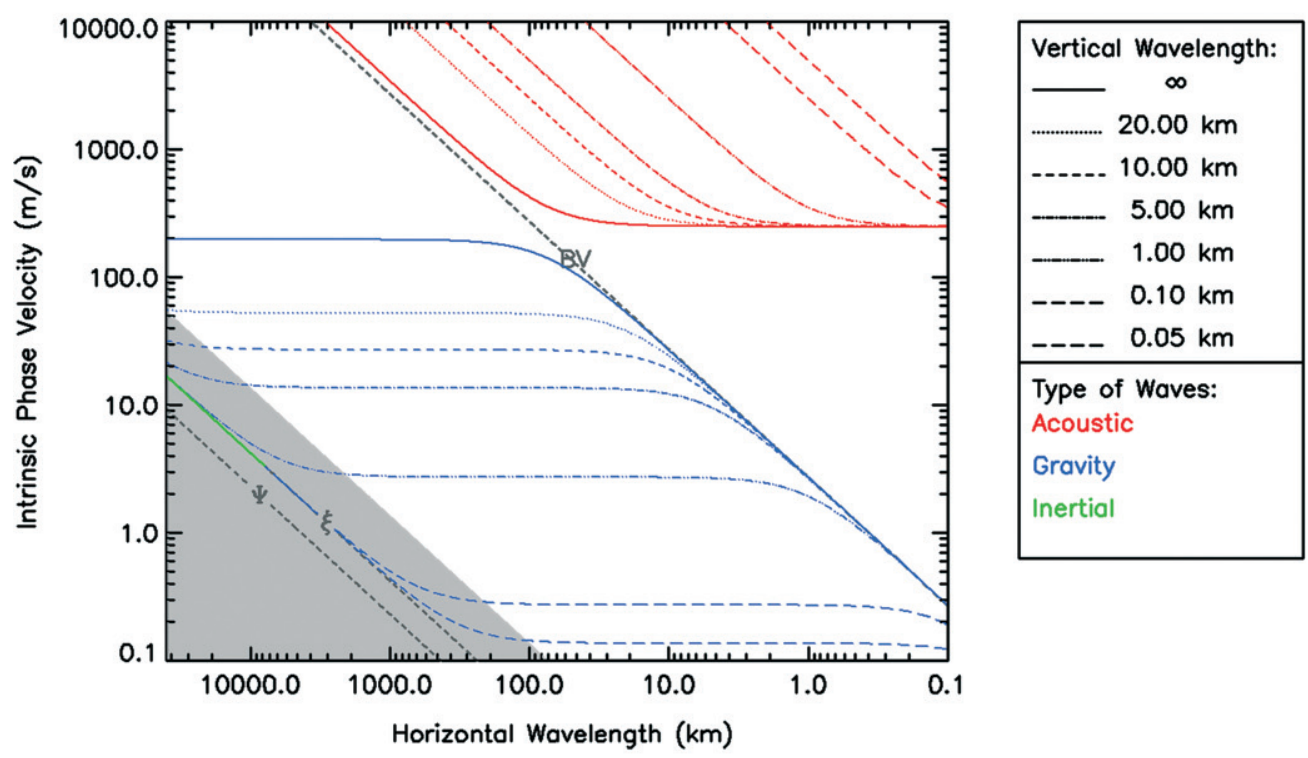

Dispersion Graph for Waves at $70.0^{\circ}$ and $47.0 \mathrm{~km}$
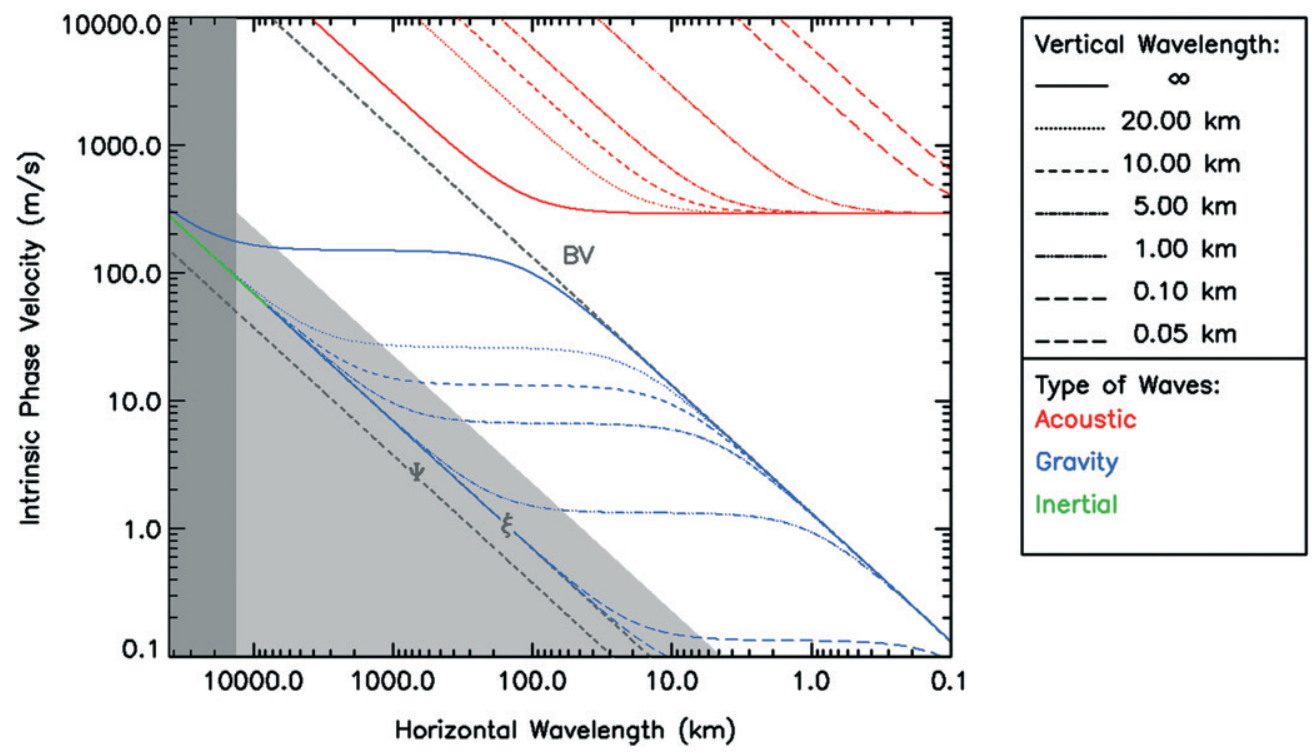

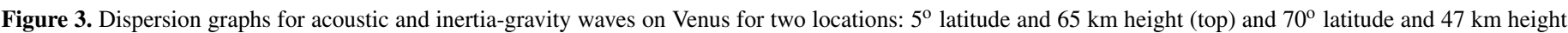

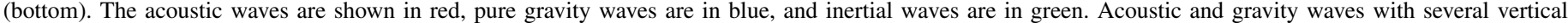

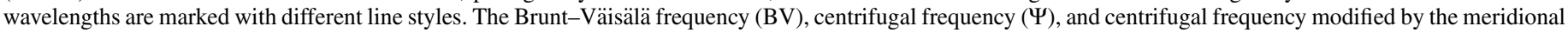

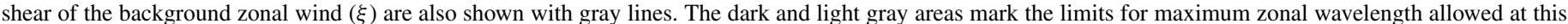

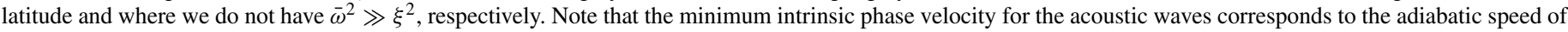
sound relative to the mean zonal flow at the marked altitude of Venus.

(A color version of this figure is available in the online journal.)

atmosphere by means of modifying the value of the centrifugal frequency $\Psi \equiv\left(u_{0} / a\right) \tan \phi$, this being a consequence of its integration in the squared frequency $\xi^{2}=2 \Psi\left(\Psi-\partial u_{0} / \partial y\right)$ (see Equations (6) and (15)). In fact, both the centrifugal frequency and the meridional shear of the wind vary significantly with latitude and, as they act together, we must study their effect directly through the squared frequency $\xi^{2}$. For simplicity, and similar to what we did for the vertical shear of the background zonal wind, we have assumed that $\xi^{2}$ (and thus $\Psi$ and $\partial u_{0} / \partial y$ ) is locally constant with latitude within the horizontal extension of the atmospheric waves, which is not generally true in the cloud region of Venus, as can be seen in Figure 4(A). In fact, the value of $\xi^{2}$ changes by several orders of magnitude $\left(10^{-12}-10^{-9} \mathrm{~s}^{-2}\right)$ from the equator to the poles at a fixed altitude, which necessarily restricts the meridional extension of inertiagravity waves that arise as a solution when $\xi^{2}$ is considered to be locally constant. Figure 4(B) exhibits the maximum meridional length permitted for waves obtained under the assumption of $\xi^{2} \neq f(y)$. In this case, we have decided to use as criterion the meridional distance needed for the squared frequency $\xi^{2}$ to double its value, i.e., $\xi_{0}^{2}+\left(d \xi^{2} / d y\right) \cdot \Delta y=2 \xi_{0}^{2}$. Two criteria can be thus used to evaluate the validity of the dispersion relations obtained under the assumption of $\xi^{2}$ being locally constant: (1) cases of inertia-gravity waves with $\bar{\omega}^{2} \gg \xi^{2}$ (see Figures 3 
Table 2

Dispersion Relation for Inertia-gravity Waves in a Cyclostrophic Atmosphere under Different Limiting Cases

\begin{tabular}{llll}
\hline \hline Approximation & \multicolumn{1}{c}{ Dispersion Relation } & Properties & Type of Wave \\
\hline$\ldots$ & $\bar{\omega} \approx \pm \sqrt{\frac{g B \cdot k_{x}^{2}+\xi^{2} \cdot\left(m^{2}+\frac{1}{4 H_{0}^{2}}\right)}{k_{x}^{2}+m^{2}+\frac{1}{4 H_{0}^{2}}}}$ & Dispersive & Inertia-gravity \\
$B=0$ & $\bar{\omega} \approx \pm \xi \cdot \sqrt{\frac{m^{2}+\frac{1}{4 H_{0}^{2}}}{k_{x}^{2}+m^{2}+\frac{1}{4 H_{0}^{2}}}}$ & Dispersive & Inertial \\
$k_{x}^{2} \ll m^{2}+\frac{1}{4 H_{0}^{2}}$ & $\bar{\omega} \approx \pm \xi= \pm \sqrt{2 \Psi^{2}-2 \Psi \frac{\partial u_{0}}{\partial y}}$ & Dispersive & \\
\hline$u_{0} \cong 0$ & $\bar{\omega} \approx \pm \frac{\sqrt{g B} \cdot k_{x}}{\sqrt{k_{x}^{2}+m^{2}+\frac{1}{4 H_{0}^{2}}}}$ & Dispersive & Gravity \\
$\{$ Lat $\}=0$ & $\bar{\omega} \approx \pm \frac{\sqrt{g B} \cdot k_{x}}{\sqrt{k_{x}^{2}+m^{2}}}$ & Dispersive & \\
$\left|k_{x}^{2}+m^{2}\right| \gg \frac{1}{4 H_{0}^{2}}$ & $\bar{\omega} \approx \pm \sqrt{g B}$ & Dispersive & \\
$k_{x}^{2} \gg m^{2}+\frac{1}{4 H_{0}^{2}}$ & &
\end{tabular}

Notes. Note that pure inertial waves cannot exist at the equator or when the atmosphere is at rest, while pure gravity waves are only possible when $B \neq 0$. Larger-scale waves are usually inertial, while pure gravity waves are the norm for smaller spatial scales.

Table 3

Dispersion Relation for Acoustic Waves under Different Limiting Cases

\begin{tabular}{|c|c|c|c|}
\hline Approximation & Dispersion Relation & Properties & Type of Wave \\
\hline & $\bar{\omega} \approx \pm \sqrt{k_{x}^{2}+m^{2}+\frac{1}{4 H_{0}^{2}}} \cdot c_{S}$ & Dispersive & Acoustic \\
\hline$\left|k_{x}^{2}+m^{2}\right| \ll \frac{1}{4 H_{0}^{2}}$ & $\bar{\omega} \approx \pm \frac{c_{S}}{2 H_{0}}$ & Dispersive Null group velocity & \\
\hline$k_{x}^{2} \ll m^{2}+\frac{1}{4 H_{0}^{2}}$ & $\bar{\omega} \approx \pm \sqrt{m^{2}+\frac{1}{4 H_{0}^{2}}} \cdot c_{S}$ & Dispersive Vertical propagation & \\
\hline$\left|k_{x}^{2}+m^{2}\right| \gg \frac{1}{4 H_{0}^{2}}$ & $\bar{\omega} \approx \pm \sqrt{k_{x}^{2}+m^{2}} \cdot c_{S}$ & Non-dispersive Horiz. propagation & \\
\hline
\end{tabular}

Notes. Note that longer acoustic waves are dispersive and tend to propagate vertically, while shorter acoustic waves are non-dispersive, propagate with the adiabatic speed of sound relative to the background zonal wind, and their propagation can be demonstrated to be practically vertical.

and 5) and (2) in the case of having $\bar{\omega}^{2} \approx \xi^{2}$, then our equations will be valid for inertia-gravity waves whose meridional length is smaller than the maximum set in Figure 4.

\section{FILTERING WAVES IN A CYCLOSTROPHIC ATMOSPHERE}

In Section 2, we introduced the tracer parameters $\left(n_{1}, n_{2}, n_{3}, n_{4}\right)$ multiplying in Equations (4a)-(4e) those terms that are key for filtering specific types of waves. In this section, we analyze the main implications of neglecting the terms associated with these tracers in the dispersion relation (Equation (13)), and then comparing the resulting dispersion relations with the generic one for acoustic and inertia-gravity waves (Equations (20a) and (20b)). We also investigate under which conditions the different simplifications can be applied to the set of equations. The relation between these tracer parameters and some important dimensionless parameters (the Mach, Richardson, and Froude numbers, as well as the aspect ratio parameter) is explored with more detail in Appendix E.

\subsection{The Hydrostatic Approximation}

The hydrostatic balance implies $D w / D t=0$ (Holton 2004), i.e., the term affected by the tracer parameter $n_{4}$ in the vertical momentum equation (Equation (4c)). Setting $n_{4}=0$ and $n_{1}=n_{2}=n_{3}=1$ in the generic dispersion relation (13) is only justified if $\bar{\omega} \ll \sqrt{g B}$. For this reason, acoustic waves $(\bar{\omega} \gg \sqrt{g B})$ and gravity waves with short horizontal wavelengths $(\bar{\omega} \stackrel{k \gg}{\longrightarrow} \sqrt{g B})$ cannot occur when the atmosphere is in hydrostatic balance. Applying the hydrostatic balance and $B+g / c_{S}^{2}=1 / H_{0}$, the dispersion relation (Equation (13)) becomes

$$
\bar{\omega}^{2}=\frac{g B \cdot k_{x}^{2}+\xi^{2} \cdot\left[m^{2}+\left(2 H_{0}\right)^{-2}\right]}{m^{2}+\left(2 H_{0}\right)^{-2}},
$$

and when compared with the dispersion relation for the inertiagravity waves (Equation (20a)), it is clear that both expressions are identical except for the term $k_{x}^{2}$ in the denominator, which is absent in Equation (22). Consequently, the inertia-gravity waves undergo a distortion under the hydrostatic approximation except when $k_{x}^{2} \ll m^{2}+\left(2 H_{0}\right)^{-2}$. As a consequence, the hydrostatic balance filters waves with horizontal scales similar to or much smaller than the vertical one and also filters the acoustic waves (what is obvious since no dispersion relation resembling Equation (20b) is obtained in this case).

\subsection{The Incompressible and Anelastic Atmospheres}

A variety of different approximations have been classically applied in order to filter the atmospheric acoustic waves (Durran 2008). One common assumption is that of the incompressible atmosphere, consisting of setting $D \rho / D t=0\left(n_{1}=n_{2}=0\right)$, thus 

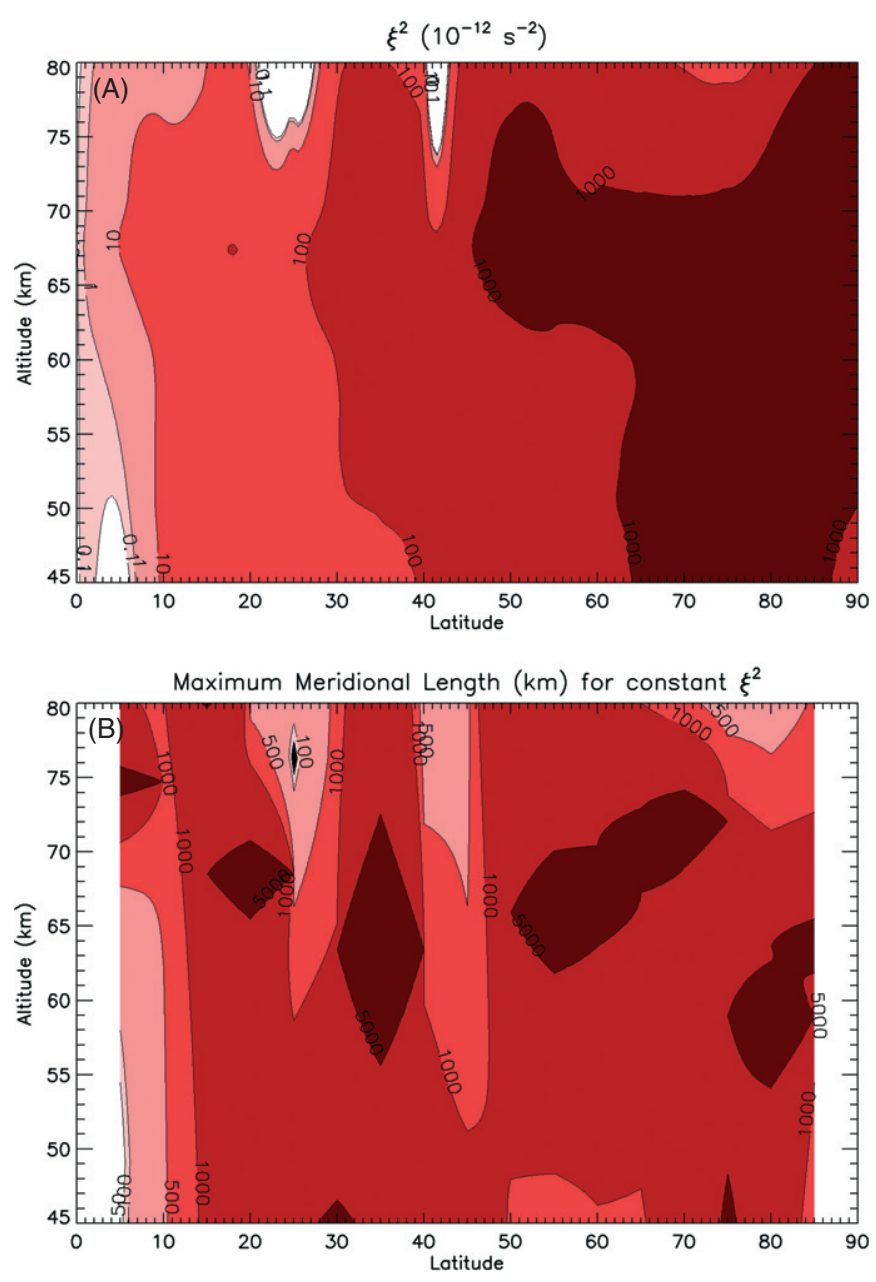

Figure 4. Profiles for the squared frequency $\xi^{2}=2 \Psi\left(\Psi-\partial u_{0} / \partial y\right)$ with units $10^{-12} \mathrm{~s}^{-2}$ (panel (A)) and maximum meridional length (in kilometers) allowed under the assumption of $\xi^{2} \neq f(y)$ (panel (B)). Note that as a consequence of the variation of the centrifugal frequency and meridional shear of the background wind, $\xi^{2}$ increases from the equator to the poles from $10^{-12} \mathrm{~s}^{-2}$ to $10^{-9} \mathrm{~s}^{-2}$. The meridional length in panel (B) corresponds to the distance required for $\xi^{2}$ to double its value. The numerical values have been extracted from our reference atmosphere (see Appendix A).

(A color version of this figure is available in the online journal.)

the atmosphere behaving as nondivergent. From Equation (13), it can be seen that applying this assumption leaves a non-null value for the term $B\left(n_{2}-n_{3}\right)$, which makes no sense since this term is spurious and does not exist when all the tracer parameters are set to 1 . As a result, the only way of applying an incompressible atmosphere is setting $n_{1}=n_{2}=n_{3}=0$ and $n_{4}=1$, which leads to the following dispersion relation:

$$
\bar{\omega}^{2}=\frac{g B \cdot k_{x}^{2}+\xi^{2} \cdot\left(m^{2}\right)}{k_{x}^{2}+m^{2}} .
$$

When compared with the generic dispersion relation (Equation (20a)), the relation (Equation (23)) clearly shows again a case of distorted inertia-gravity waves, with several terms missing $\left(1 / 4 H_{0}^{2}\right.$ and a $k_{x}^{2}$ in the numerator). Thus, the incompressible atmosphere approximation severely affects the inertia-gravity waves, an unwanted result if our intention is to filter the acoustic waves and leave inertia-gravity waves unaltered.

Alternatively, another frequent assumption is considering that the medium is anelastic, i.e., assuming that $\partial \rho / \partial t=0$ in the continuity equation. However, in the case of a cyclostrophic atmosphere, the advection term $u_{0}(\partial \rho / \partial x)$ remaining in the continuity equation (Equation (4d)) extremely complicates the procedure for solving the wave disturbance equations. Alternatively, we propose considering that the atmosphere is intrinsically anelastic, i.e., that the atmosphere behaves as anelastic relative to the background wind (note that this is equivalent to assuming an almost incompressible atmosphere, except for the non-negligible vertical gradient of the basic-state density). This procedure for filtering the acoustic waves is quite similar to the one applied for Venus by Leroy \& Ingersoll (1995, see their Equation (1d)). In this case, we set $n_{2}=n_{3}=0$ and $n_{1}=n_{4}=1$, obtaining the next dispersion relation:

$$
\bar{\omega}^{2}=\frac{g B \cdot k_{x}^{2}+\xi^{2} \cdot\left[m^{2}+\left(2 H_{0}\right)^{-2}\right]}{k_{x}^{2}+m^{2}+\left(2 H_{0}\right)^{-2}},
$$

which is exactly the dispersion relation for inertia-gravity waves (Equation (20a)). We can conclude that applying this intrinsically anelastic atmosphere approximation permits one to filter the acoustic waves while keeping the inertia-gravity waves unaltered.

\subsection{The Boussinesq Approximation and Hydrostatic Balance}

In Sections 5.1 and 5.2, we showed that the approximations of intrinsic-anelasticity and hydrostatic balance allow one to filter the acoustic waves and all high-frequency waves, respectively. This is achieved by removing the terms in the continuity and vertical momentum equations that contain the tracer parameters $n_{2}, n_{3}$, and $n_{4}$. If we set $n_{1}=0$, we would obtain an atmosphere with null vertical variation for the density

$$
\frac{\partial \rho_{0}}{\partial z}=0 \Leftrightarrow \frac{1}{H_{0}} \equiv-\frac{1}{\rho_{0}} \frac{\partial \rho_{0}}{\partial z}=0 \Leftrightarrow n_{1}=0 .
$$

At this stage, we can explore the effect of canceling all the tracer parameters (i.e., $n_{1}=n_{2}=n_{3}=n_{4}=0$ ). Applying this to the system of Equations ((4a)-(4d)) yields

$$
\begin{gathered}
\frac{\partial u^{\prime}}{\partial t}+u_{0} \frac{\partial u^{\prime}}{\partial x}+\frac{\partial}{\partial x}\left(\frac{P^{\prime}}{\rho_{0}}\right)-\Psi \cdot v^{\prime}+\frac{\partial u_{0}}{\partial y} v^{\prime}=0 \\
\frac{\partial v^{\prime}}{\partial t}+u_{0} \frac{\partial v^{\prime}}{\partial x}+2 \Psi \cdot u^{\prime}=0 \\
\frac{\partial}{\partial z}\left(\frac{P^{\prime}}{\rho_{0}}\right)-g \cdot \Theta^{\prime}=0 \\
\frac{\partial u^{\prime}}{\partial x}+\frac{\partial v^{\prime}}{\partial y}+\frac{\partial w^{\prime}}{\partial z}=0
\end{gathered}
$$

which is the set of equations that we would expect after jointly applying the Boussinesq approximation and hydrostatic balance, and it can also be demonstrated that the axis rotation made in Section 2 is no longer needed when the Boussinesq approximation is used (see Appendix B in Peralta et al. 2014).

If we also set $n_{1}=n_{2}=n_{3}=n_{4}=0$ in Equation (13), it is straightforward to obtain the following dispersion relation:

$$
\bar{\omega}^{2}=\frac{g B \cdot k_{x}^{2}+\xi^{2} \cdot\left(m^{2}\right)}{m^{2}},
$$

which is similar to the dispersion relation for the inertia-gravity waves (Equation (20a)), except for the absence of $k_{x}^{2}$ and $1 / 4 H_{0}^{2}$ in some of its terms. A straightforward interpretation is that 
the dispersion relation (Equation (27)) describes again inertiagravity waves distorted by the different assumptions already applied (hydrostatic and Boussinesq approximations), which are clearly unable to filter acoustic and inertia-gravity waves at the same time.

\subsection{Wave Filtering on Cyclostrophic and Geostrophic Atmospheres}

Most of the atmospheric simplifications introduced in this section have the same effect of filtering waves on the terrestrial and Venusian conditions. For instance, in both geostrophic and cyclostrophic regimes, the hydrostatic approximation $(D w / D t=0)$ identically filters waves with horizontal scales similar to or much smaller than vertical ones and filters the acoustic waves (Norbury \& Roulstone 2002), and the Boussinesq and incompressibility approximations filter acoustic waves but lead to distorted gravity waves (Durran 2008). On the other hand, the anelastic approximation $(\partial \rho / \partial t=0)$ effectively filters acoustic waves while leaving the gravity waves unaltered; to obtain the same result, we showed that it should be applied differently by setting $\partial \rho / \partial t+u_{0} \partial \rho / \partial x=0$, i.e., by assuming that the atmosphere is anelastic relative to the background zonal wind or intrinsically anelastic.

\section{DISCUSSION}

As stated in the introduction, atmospheric waves are expected to play a crucial role in the generation and maintenance of superrotation. In the case of Venus, its atmosphere is rich in wave activity at many vertical levels and a wide range of spatial scales (Seiff et al. 1992). Thus, developing a systematic and easy way to classify these waves attending to their measurable characteristics seems to be a crucial task towards a definitive explanation of the mechanisms for superrotation and general circulation. The dispersion relations (Equations (20a) and (20b)) derived for a generic planet with an atmosphere in the cyclostrophic regime can be used to obtain the dispersion graphs (see Figure 3) for diverse regions of the atmosphere, provided that we know the values of the atmospheric static stability, gravity acceleration, speed of sound, and the background zonal wind. For Venus, this can be easily carried out by making use of the reference atmosphere presented in this work (see Appendix A).

In this sense, we can apply our dispersion graphs to classify the horizontal mesoscale waves found at different vertical levels of the Venus atmosphere with the imaging spectrometer VIRTIS-M and the Venus Monitoring Camera (VMC) on board the spacecraft Venus Express (Peralta et al. 2008; Piccialli et al. 2014). Basically, three levels of the Venus cloud region were sensed with these instruments while seeking atmospheric waves: the upper cloud at about $66 \mathrm{~km}$ in height with dayside $380 \mathrm{~nm}$ images, the base of the upper cloud at $61 \mathrm{~km}$ with dayside $980 \mathrm{~nm}$ observations, and the lower clouds with nightside images taken at $1.74 \mu \mathrm{m}$ corresponding to the surface thermal radiation attenuated by clouds between 44 and $48 \mathrm{~km}$ (Peralta et al. 2008). In the case of the VIRTIS-M observations, these were made covering the southern hemisphere and both the horizontal wavelength and intrinsic phase velocities were measured when possible (Peralta et al. 2008). On the other hand, wave observations by VMC were made during the periapsis of the orbit, thus covering only the northern hemisphere and inhibiting the estimate of the corresponding phase velocities as a consequence of the high speed of the spacecraft (Piccialli et al. 2014). Figure 5 displays dispersion graphs of the upper and lower clouds of Venus as well as the position of the horizontal mesoscale waves found in the Venus Express mission:

One of the main conclusions we can extract is that our dispersion graphs confirm the first hypotheses identifying these mesoscale waves apparent in the clouds with non-dispersive gravity waves. This conclusion is direct for those cases with both horizontal wavelengths and intrinsic phase velocities measured (asterisks). For the northern waves identified in the VMC images, only the wavelength was measured (Piccialli et al. 2014) and we cannot be sure whether we are dealing with acoustic or gravity waves. If these waves are acoustic, they are probably thermally induced compression waves, generated when a parcel of air is heated, and then pressure increases within the air parcel, causing it to expand and causing the adjacent air to be compressed, thus generating a compression wave (Nicholls $\&$ Pielke 2000). Both the density and temperature increase in the compressed air, which would explain the cloud patterns seen in the VMC images. On the other hand, if we assume to be observing gravity waves as in the southern hemisphere, our dispersion graph allows one to predict an upper limit to the intrinsic phase velocity for each horizontal wavelength. Regarding the waves apparent in the lower clouds (see Figure 5, panel below), it is worth noting that, except for one case, the predicted vertical wavelengths have values below $10 \mathrm{~km}$, consistent with the vertical extension of the static stability region where these waves are confined (Peralta et al. 2008).

Finally, we would like to discuss the applicability of our results to the increasing catalog of exoplanets discovered to date (Schneider et al. 2011). In principle, the theory developed for atmospheric waves can be applied to both "planet-wide" cyclostrophic regimes (as in the cases of Venus, Titan, and other slowly rotating exoplanets yet to be discovered) and also "locally" considering that Coriolis terms vanish near the equator or on smaller scales such as storm systems and eddies (de Pater \& Lissauer 2001). The possibility of a local validity for the cyclostrophic balance at the equator is especially relevant as both recent observations (Faigler et al. 2013) and atmospheric circulation models (Showman \& Polvani 2011) display equatorial superrotation in tidally locked exoplanets with short periods. On the other hand, we can make an attempt to estimate an approximate number of exoplanets with "planetwide" cyclostrophic regimes by approximating the values of the Rossby number, which are provided by the expression $R o=V /(f \cdot L)$, where $V$ is the magnitude of the atmospheric winds, $f=2 \Omega \sin \phi$ is the Coriolis factor (in this case, taken at the latitude $\phi=30), \Omega$ is the rotation rate of the planet, and, for the distance $L$, we assume the value of the exoplanet's radius. Given that the rotation rate is still unknown for exoplanets, we restricted this to those cases that are expected to be tidally locked to their star (i.e., those whose orbital semi-major axis is lower than the tidal locking radius, $\left.R_{\text {Tidal }}\right)$ and for which the orbital period can be estimated to match the rotation rate:

$$
R_{\text {Tidal }}=0.0483 \cdot \sqrt[6]{\frac{\tau_{\text {Star }} \cdot M_{\text {Star }}^{2}}{\rho_{\text {Planet }}}},
$$

where $R_{\text {Tidal }}$ is given in $\mathrm{AUs}, \tau_{\mathrm{Star}}$ is the age of the star in years, $M_{\text {Star }}$ is the mass of the star in solar masses, and $\rho_{\text {Planet }}$ is the density of the exoplanet in tons $\mathrm{dm}^{-3}$. This formula is a proxy for the one originally suggested by Peale (1977) and later used in other works (Kasting et al. 1993). Table 4 exhibits the exoplanets which are the most serious candidates for having a cyclostrophic regime among those listed on the 
Dispersion Graph for Waves at $70.0^{\circ}$ and $65.0 \mathrm{~km}$
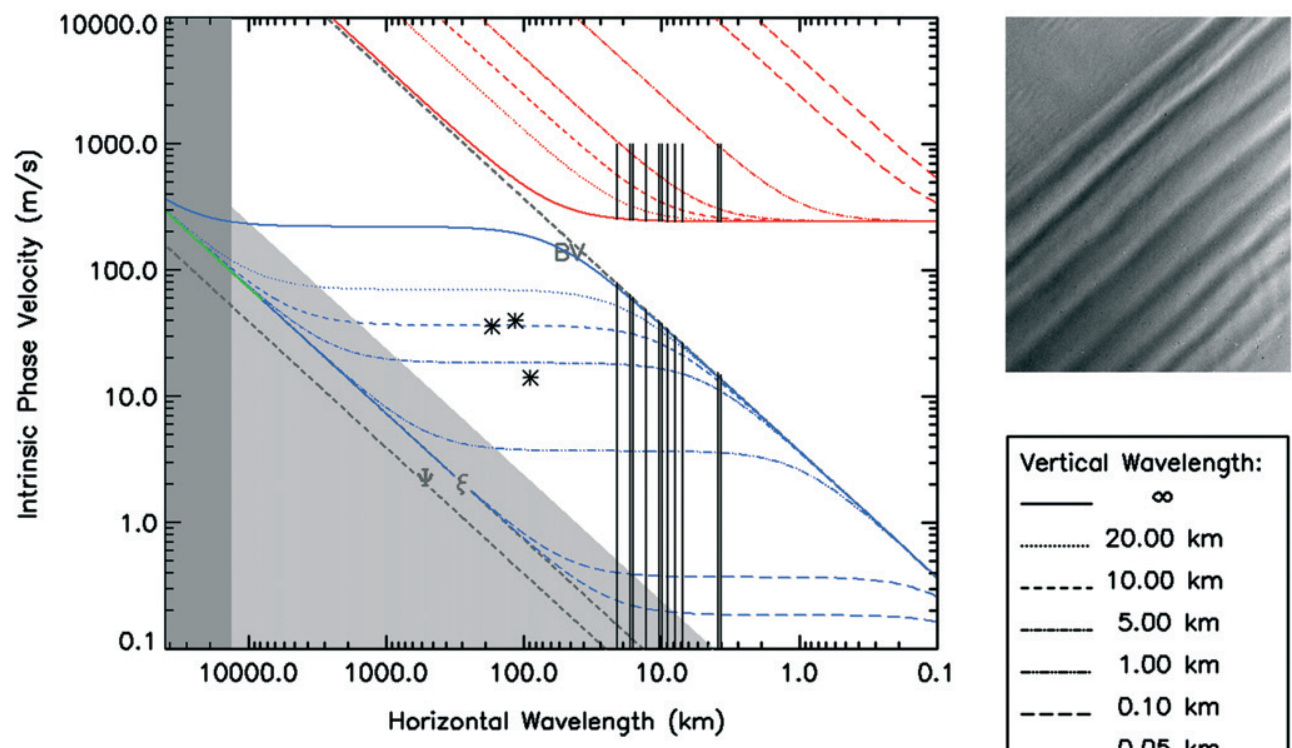

Vertical Wavelength:
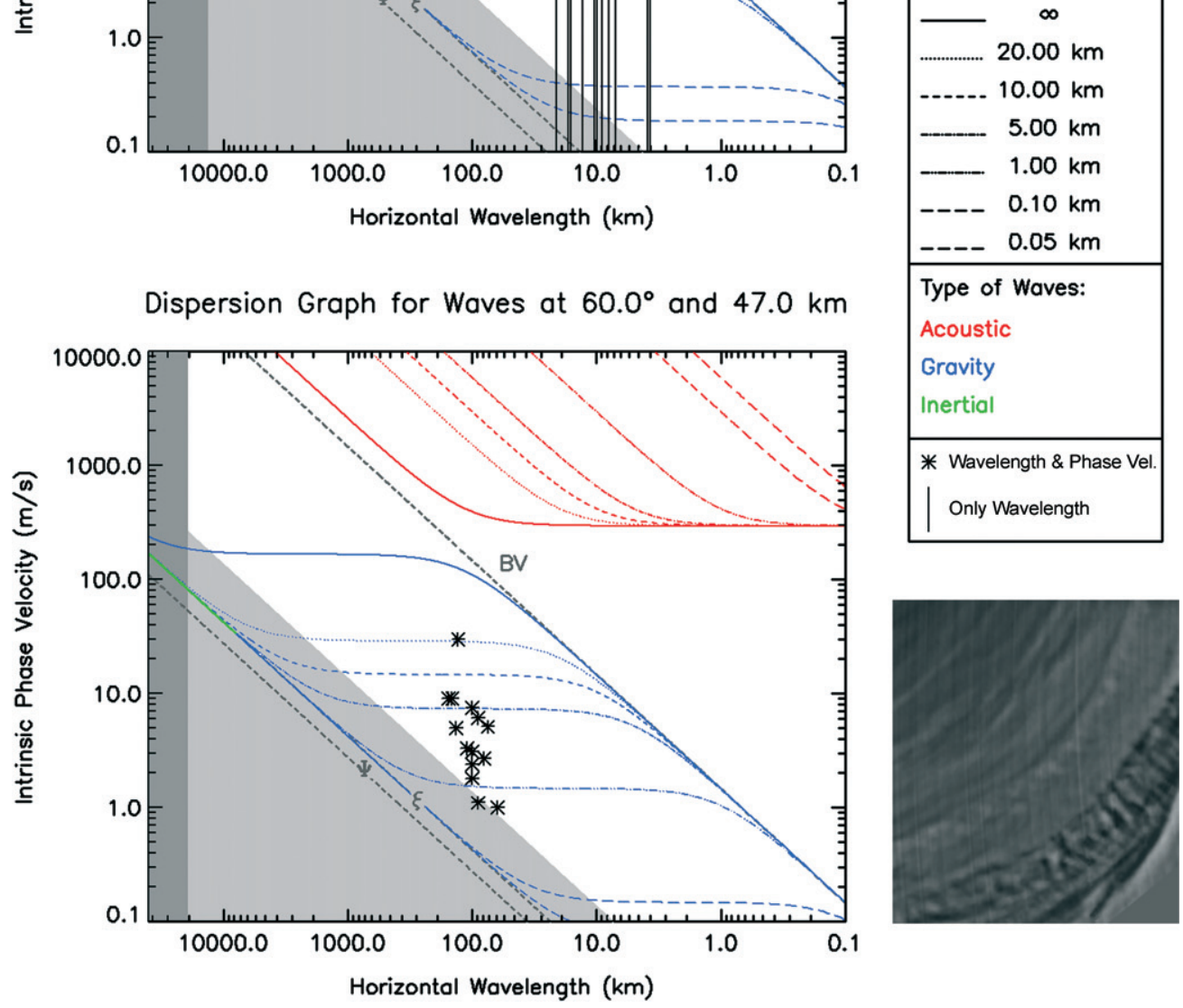

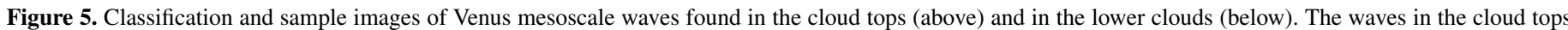

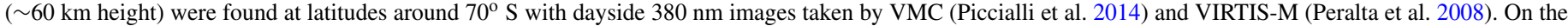

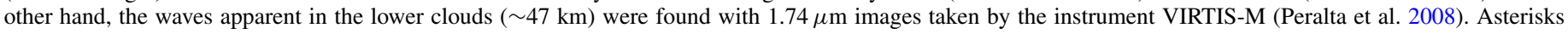

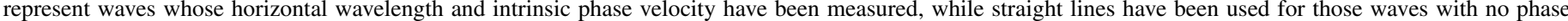

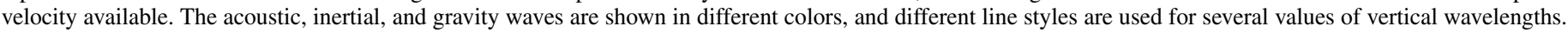

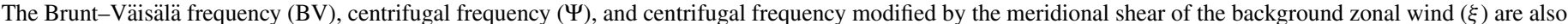

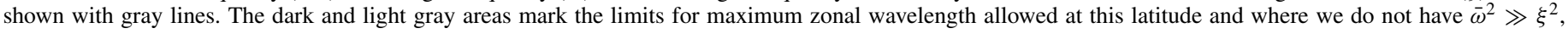
respectively.

(A color version of this figure is available in the online journal.)

Web site http://exoplanet.eu/. To this aim, we have calculated the Rossby number for two possible orders of magnitude in the atmospheric wind $\left(100\right.$ and $\left.1000 \mathrm{~m} \mathrm{~s}^{-1}\right)$, with the highest value having been taken from Doppler estimates in exoplanets (Snellen et al. 2010). For reference, a geostrophic regime is characterized by $R o \ll 1$, while a cyclostrophic system has $R o \gg 1$ (Schubert 1983). Assuming the same criteria for Titan (Bird et al. 2005) and Venus (Schubert 1983), we will consider $L \sim$ radius $\left(L_{T}=2575 \mathrm{~km}\right.$ and $\left.L_{V}=6052 \mathrm{~km}\right)$, wind velocities of about $100 \mathrm{~m} \mathrm{~s}^{-1}$, and their own rotation rates
$\left(\Omega_{T}=4.56 \times 10^{-6} \mathrm{rad} \mathrm{s}^{-1} \& \Omega_{V}=2.99 \times 10^{-7} \mathrm{rad} \mathrm{s}^{-1}\right)$. Hence, the Rossby numbers for Titan and Venus are $R o \approx 8$ and $R o \approx 55$, respectively.

\section{CONCLUSIONS}

In slowly rotating planets, the Coriolis terms can be neglected and their atmosphere can be described by a cyclostrophic regime instead of a geostrophic one; then, the pressure gradient force is balanced by the centrifugal force. Under reasonable 
Table 4

List of Tidally Locked Exoplanets That are Candidates for Having an Atmosphere in Cyclostrophic Balance

\begin{tabular}{|c|c|c|c|c|c|c|c|c|c|}
\hline \multicolumn{2}{|c|}{ Star } & \multicolumn{8}{|c|}{ Exoplanet } \\
\hline $\begin{array}{l}\text { Mass } \\
\left(M_{\text {Sun }}\right)\end{array}$ & $\begin{array}{l}\text { Age } \\
(\mathrm{Gyr})\end{array}$ & Name & $\begin{array}{c}\text { Mass } \\
\left(M_{\text {Jup }}\right)\end{array}$ & $\begin{array}{l}\text { Radii } \\
\left(R_{\text {Jup }}\right)\end{array}$ & $\begin{array}{c}\text { Orbit Radii } \\
\text { (AU) }\end{array}$ & $\begin{array}{c}\text { Orbit Period } \\
\text { (days) }\end{array}$ & $\begin{array}{l}\text { Tidal Lock } \\
\text { (AU) }\end{array}$ & $\begin{array}{c}R \mathrm{o} \\
\left(10^{2} \mathrm{~m} \mathrm{~s}^{-1}\right)\end{array}$ & $\begin{array}{c}R \mathrm{Ro} \\
\left(10^{3} \mathrm{~m} \mathrm{~s}^{-1}\right)\end{array}$ \\
\hline 0.95 & 8 & Kepler-11 g & 0.95 & 0.33 & 0.46 & 118 & 1.17 & 7.1 & 71 \\
\hline 1.1 & 7.5 & Kepler-87 c & 0.02 & 0.55 & 0.66 & 192 & 3.36 & 6.9 & 69 \\
\hline 1.165 & 3.44 & Kepler-79 e & 0.013 & 0.31 & 0.39 & 81 & 2.77 & 5.1 & 51 \\
\hline 0.95 & 8 & Kepler-11 f & 0.006 & 0.22 & 0.25 & 46 & 2.54 & 4.1 & 41 \\
\hline 0.98 & 7.63 & HD 80606 b & 3.94 & 0.92 & 0.45 & 111 & 2.82 & 2.4 & 24 \\
\hline 1.079 & 6.3 & Kepler-68 c & 0.015 & 0.08 & 0.09 & 9 & 1.23 & 2.3 & 23 \\
\hline 1.113 & 6.923 & Kepler-36 b & 0.014 & 0.13 & 0.11 & 13 & 2.42 & 2.1 & 21 \\
\hline 0.99 & 4 & CoRoT-9 b & 0.84 & 0.94 & 0.41 & 95 & 2.76 & 2.0 & 20 \\
\hline 1.25 & 3.9 & Kepler-89 e & 0.04 & 0.55 & 0.30 & 54 & 3.27 & 1.9 & 19 \\
\hline 1.1 & 7.5 & Kepler-87 b & 1.02 & 1.21 & 0.47 & 115 & 3.14 & 1.9 & 19 \\
\hline 1.165 & 3.44 & Kepler-79 c & 0.05 & 0.33 & 0.19 & 27 & 2.36 & 1.6 & 16 \\
\hline 0.95 & 8 & Kepler-11 d & 0.02 & 0.28 & 0.16 & 22 & 2.61 & 1.6 & 16 \\
\hline 0.95 & 8 & Kepler-11 e & 0.02 & 0.40 & 0.19 & 32 & 2.89 & 1.5 & 15 \\
\hline 1.038 & 1 & Kepler-66 b & 0.31 & 0.25 & 0.13 & 18 & 1.15 & 1.4 & 14 \\
\hline 0.95 & 8 & Kepler-11 b & 0.006 & 0.16 & 0.09 & 10 & 2.12 & 1.3 & 13 \\
\hline 0.865 & 1 & Kepler-67 b & 0.31 & 0.26 & 0.12 & 15 & 1.13 & 1.2 & 12 \\
\hline 1.25 & 2.9 & Kepler-65 d & 0.006 & 0.14 & 0.08 & 8 & 1.60 & 1.2 & 12 \\
\hline 0.95 & 8 & Kepler-11 c & 0.009 & 0.26 & 0.11 & 13 & 2.48 & 1.0 & 10 \\
\hline
\end{tabular}

Notes. Data are taken from http://exoplanet.eu/. Titan and Venus are $R o \approx 8$ and $R o \approx 55$, respectively. Geostrophic regimes occur for $R o \ll 1$ while the atmosphere is cyclostrophic when $R o \gg 1$ (Schubert 1983).

assumptions, we have deduced, for the first time, analytical solutions for acoustic and inertia-gravity waves for a cyclostrophic atmosphere, simultaneously considering the metric terms for the centrifugal force and the meridional shear of the background wind. The corresponding dispersion relations are of special interest for the classification of the numerous waves found with remote sensing data and whose nature is still unclear. Furthermore, they can help one to restrict the possible candidates for the periodicities observed in many atmospheric parameters and to characterize the wave amplitude, structure, and direction of propagation once its nature has been determined.

Acoustic and inertia-gravity waves have been found to be solutions of the equations describing a cyclostrophic atmosphere, and we checked that their properties are identical to those of their geostrophic counterparts. It has also been shown that the centrifugal force can be redefined as a centrifugal frequency that plays a role similar to the Coriolis factor on the geostrophic case, and this centrifugal frequency determines a lower limit to the intrinsic frequencies for gravity waves. The meridional shear of the mean zonal flow has been shown to be a modifier of the centrifugal frequency by restricting the region where inertiagravity waves are possible in the dispersion graph. As a result of the prevailing cyclostrophic regime, inertial waves are found to be possible on a cyclostrophic regime, and their existence is subject to an inertial stability defined in terms of the difference between the centrifugal frequency and the meridional shear of the background wind.

Acoustic and inertia-gravity waves have amplitudes that decrease exponentially with height. Large-scale acoustic waves are clearly dispersive, have intrinsic frequencies that do not depend on the wavenumber, and they have a high phase velocity and a null group velocity, so they cannot transport energy or momentum. For very small wavelengths, we recover the classical dispersion relation for acoustic waves, which are nondispersive and with an intrinsic phase velocity equal to the adiabatic speed of sound. Concerning the inertia-gravity waves, we found that in the case of short horizontal wavelengths, null background wind, or in the equatorial region, only pure gravity waves are possible. For large horizontal wavelengths and/or null static stability, the waves are inertial, dispersive, and acquire large horizontal phase speeds.

Additionally, some of the classical atmospheric approximations have been examined in order to determine their effect on the wave solutions. This helps one to determine their role in filtering waves and it is also crucial to improve and simplify, for example, current GCMs for Venus and Titan. It has been found that, as in the geostrophic case, the hydrostatic approximation filters all the high-frequency waves, while an incompressible atmosphere allows filtering the acoustic waves but severely modifies the inertia-gravity waves. We have also demonstrated that applying an intrinsically anelastic atmosphere approximationassuming that the atmosphere behaves as anelastic but only when observed relative to the background wind - the acoustic waves can be successfully filtered out, while the inertia-gravity waves remain unchanged.

Finally, we have applied our results for a systematic classification of the mesoscale waves that are apparent on the clouds at different vertical levels of Venus's atmosphere. To accomplish this, a reference model atmosphere of Venus has been created by combining atmospheric data from previous missions (Venus International Reference Atmosphere (VIRA)) with recent data from Venus Express, which allowed us to obtain dispersion relations for waves between the equator and $85^{\circ}$ and altitudes between 45 and $80 \mathrm{~km}$ (see Appendix A). We confirm that these waves are non-dispersive gravity waves, while those found in the lower clouds have vertical wavelengths compatible with the vertical extension of the stability region where they are propagating.

J. Peralta acknowledges support from the Portuguese Fundação para a Ciência e a Tecnologia (FCT, grant reference: SFRH/BPD/63036/2009), from the Spanish MICINN 
for funding support through the CONSOLIDER program "ASTROMOL" CSD2009-00038, and also funding through project AYA2011-23552. J.P. and D.L. acknowledge FCT funding through project grants POCI/CTE-AST/110702/2009, the Pessoa-PHC programme, and project PEst-OE/FIS/UI2751/ 2011. A.P. acknowledges funding from the European Union Seventh Framework Programme (FP7/2007-2013) under grant agreement No. 246556. The authors are very grateful for the invaluable revision carried out by the anonymous reviewer.

\section{APPENDIX A}

\section{REFERENCE ATMOSPHERE AND SCALE ANALYSIS FOR VENUS}

In order to simplify as much as possible the equations to be used for the deduction of the waves in the cyclostrophic atmosphere of Venus, a scale analysis of the primitive equations is required. To describe a standard atmosphere, primitive momentum, continuity, and thermodynamic equations in local east-north-up (ENU) coordinates were chosen (Holton 2004). It is important to note, however, that these coordinates only behave like genuine Cartesian ones over small spatial ranges in latitude (thus they are generally suitable for acoustic- and inertia-gravity waves):

$$
\begin{gathered}
\frac{D u}{D t}=-\frac{1}{\rho} \frac{\partial P}{\partial x}+\frac{u v}{r} \tan \phi-\frac{u w}{r}+2 \Omega \cdot v \sin \phi \\
-2 \Omega \cdot w \cos \phi+\mathrm{Fr}_{x}, \\
\frac{D v}{D t}=-\frac{1}{\rho} \frac{\partial P}{\partial y}-\frac{u^{2}}{r} \tan \phi-\frac{v w}{r}-2 \Omega \cdot u \sin \phi+\mathrm{Fr}_{y}, \\
\frac{D w}{D t}=-\frac{1}{\rho} \frac{\partial P}{\partial z}-g+\frac{u^{2}+v^{2}}{r}+2 \Omega \cdot u \cos \phi+\mathrm{Fr}_{z}, \\
\frac{D \rho}{D t}=-\rho\left(\frac{\partial u}{\partial x}+\frac{\partial v}{\partial y}+\frac{\partial w}{\partial z}\right), \\
\frac{D s}{D t}=\frac{Q}{T}, \quad \text { with } s=C_{P} \ln \theta,
\end{gathered}
$$

where $(u, v, w)$ are the zonal, meridional, and vertical components of the wind; $P$ is the atmospheric pressure; $\rho$ is the density; $g$ is the gravity acceleration on Venus; $\Omega$ is the solid planet rotation rate; $\phi$ is the latitude; Fr is the molecular friction; $r=a+z$, with $z$ being the altitude over the planet surface and $a$ the Venus radius; $s$ is the entropy; and $\theta$ is the potential temperature. We will assume that Venus's radius $a$ is much larger than the altitude over the surface $z$, so we can consider that $r \cong a$. Moreover, the molecular friction is about $10^{-12} \mathrm{~m} \mathrm{~s}^{-2}$ and can be neglected for all the motions except the smallest scale turbulent ones (Piccialli 2010). Finally, considering that the atmospheric motions are adiabatic $Q=0$ in the cloud region of Venus (Tellmann et al. 2009), and redefining the potential temperature as $\Theta \equiv \ln \theta$, we will then have

$$
\frac{D u}{D t}=-\frac{1}{\rho} \frac{\partial P}{\partial x}+\frac{u v}{a} \tan \phi-\frac{u w}{a}+2 \Omega \cdot(v \sin \phi-w \cos \phi),
$$

$$
\begin{gathered}
\frac{D v}{D t}=-\frac{1}{\rho} \frac{\partial P}{\partial y}-\frac{u^{2}}{a} \tan \phi-\frac{v w}{a}-2 \Omega \cdot u \sin \phi \\
\frac{D w}{D t}=-\frac{1}{\rho} \frac{\partial P}{\partial z}-g+\frac{u^{2}+v^{2}}{a}+2 \Omega \cdot u \cos \phi
\end{gathered}
$$
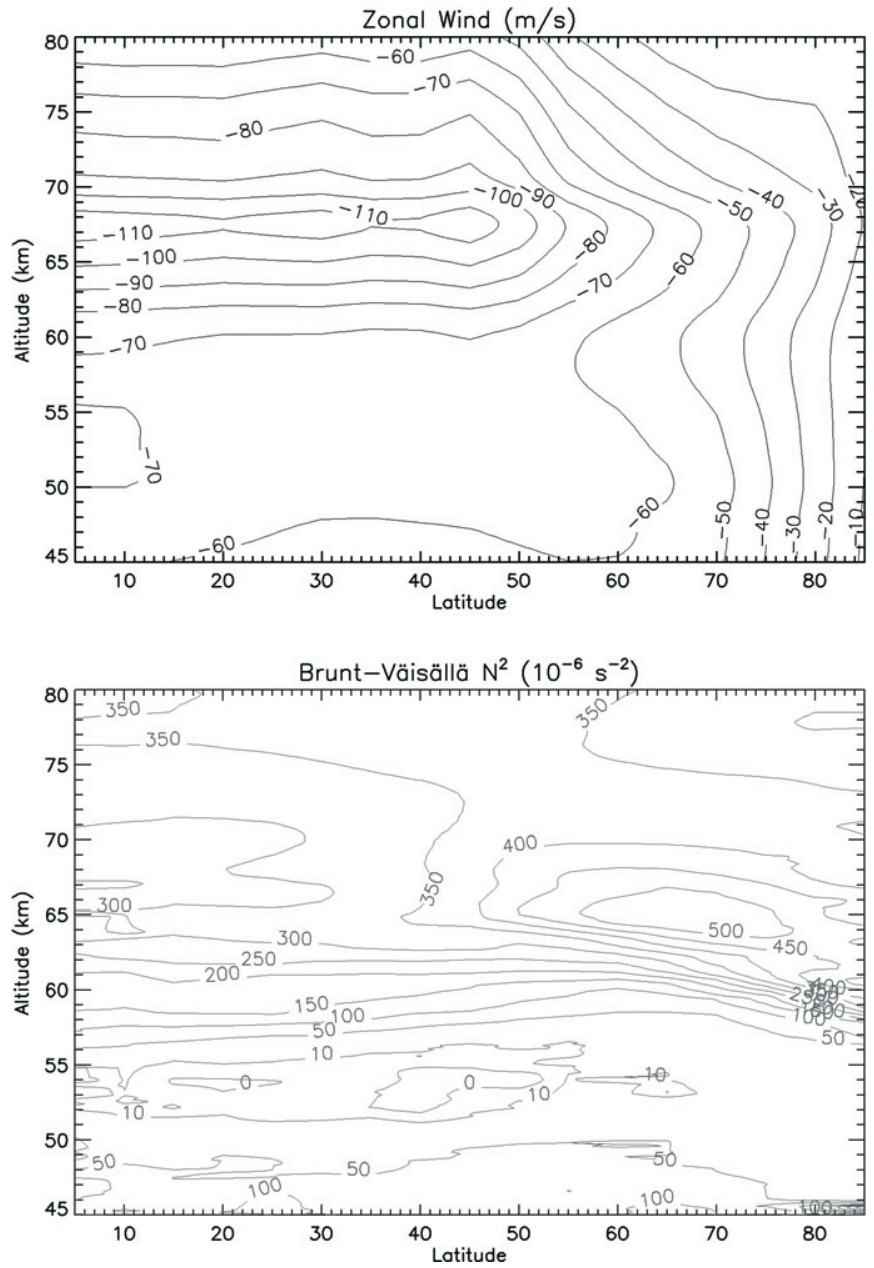

Figure 6. Zonal wind (top) and atmospheric Brunt-Väisälä frequency (bottom) for the model reference atmosphere. The zonal wind has been interpolated using data from cloud tracking (Hueso et al. 2012) and the vertical profiles obtained during the Veneras and Pioneer Venus missions (Gierasch et al. 1997). The Brunt-Väisällä frequency comes from results by VEX-VeRA (Tellmann et al. 2009).

$$
\begin{gathered}
\frac{D \rho}{D t}=-\rho\left(\frac{\partial u}{\partial x}+\frac{\partial v}{\partial y}+\frac{\partial w}{\partial z}\right), \\
\frac{D \Theta}{D t}=0 .
\end{gathered}
$$

In order to estimate orders of magnitude for performing a deeper scale analysis to the momentum equations (Equations (A2a)-(A2c)), we constructed a simple reference model for the atmosphere of Venus at the level of the clouds, combining and interpolating data from the Pioneer Venus mission (Seiff et al. 1985) and recent results from Venus Express (Tellmann et al. 2009; Piccialli 2010; Hueso et al. 2012). This reference model is axisymmetric, covering latitudes between the equator and the pole with steps of $5^{\circ}$, and heights between 45 and $80 \mathrm{~km}$ with steps of $100 \mathrm{~m}$. Typical values of zonal wind and Brunt-Väisälä frequency are displayed in Figure 6. The static stability and pressure data come from Venus Express radio occultation measurements (Tellmann et al. 2009; Piccialli 2010), while the density, temperature, and heat capacities at constant pressure and volume were extracted from VIRA. The zonal wind was the result of an interpolation using the meridional profiles at two altitudes extracted from cloudtracking analysis of Venus Express/VIRTIS images (Hueso et al. 
Table 5

Order of Magnitude for Some of the Most Relevant Atmospheric Parameters for Venus and Earth (Piccialli 2010)

\begin{tabular}{lccc}
\hline \hline Scale & Symbol & Venus & Earth \\
\hline Vertical scale $(\mathrm{m})$ & $H$ & $10^{4}$ & $10^{3}$ \\
Zonal velocity $\left(\mathrm{m} \mathrm{s}^{-1}\right)$ & $U$ & $10^{2}$ & 10 \\
Meridional velocity $\left(\mathrm{m} \mathrm{s}^{-1}\right)$ & $V$ & 10 & 10 \\
Vertical Velocity $\left(\mathrm{m} \mathrm{s}^{-1}\right)$ & $W$ & 1 & $10^{-2}$ \\
Radius $(\mathrm{m})$ & $a$ & $6 \cdot 10^{6}$ & $6 \cdot 10^{6}$ \\
Rotation rate $\left(\mathrm{rad} \mathrm{s}^{-1}\right)$ & $\Omega$ & $10^{-7}$ & $10^{-5}$ \\
Acceleration of gravity $\left(\mathrm{m} \mathrm{s}^{-2}\right)$ & $g$ & 8.8 & 9.8 \\
Timescale (s) & $T$ & $10^{6}$ & $10^{5}$ \\
\hline
\end{tabular}

2012) combined with the vertical profiles extracted with Pioneer Venus and Venera probes at specific latitudes (Seiff et al. 1985; Gierasch et al. 1997).

Table 5 shows the typical scales for Venus at the region of the clouds (Schubert 1983) compared to the Earth (Holton 2004):

Using our reference atmosphere for Venus, we can consider the maximum value within the cloud region for all the terms appearing on the right-hand side of Equations ((A2a) and (A2c)). From Table 6 , the maximum magnitude is $10^{-3}$ for the zonal and meridional momentum equations and $10^{1}$ for the vertical momentum. As the advection terms within the material derivative $D / D t$ are not sufficiently constrained from measurements, they were not evaluated for the scale analysis.

Thus, according to Table 6 , we will make the following assumptions to apply the scale analysis to the momentum equations.

1. Only those terms with the maximum scale will be considered.

2. All the advection terms will be included.

3. In order to study the atmospheric waves of Venus with a set of equations similar to the momentum equations for the terrestrial case, the minor metric term $(u v / a) \tan \phi$ will be included in the zonal equation.

We finally obtain

$$
\begin{gathered}
\frac{D u}{D t}=-\frac{1}{\rho} \frac{\partial P}{\partial x}+\frac{u v}{a} \tan \phi, \\
\frac{D v}{D t}=-\frac{1}{\rho} \frac{\partial P}{\partial y}-\frac{u^{2}}{a} \tan \phi, \\
\frac{D w}{D t}=-\frac{1}{\rho} \frac{\partial P}{\partial z}-g, \\
\frac{D \rho}{D t}=-\rho\left(\frac{\partial u}{\partial x}+\frac{\partial v}{\partial y}+\frac{\partial w}{\partial z}\right), \\
\frac{D \Theta}{D t}=0 .
\end{gathered}
$$

\section{APPENDIX B}

\section{APPLICABILITY OF THE METHOD OF MULTIPLE SCALES ON VENUS}

The method of multiple scales in height allows one to simplify the procedure to solve the wave equations under the combined effect of vertical and horizontal shear of the background wind (Boyd 1978). When this method is valid, we

\begin{tabular}{|c|c|c|c|c|c|}
\hline \multicolumn{2}{|c|}{ Zonal Equation } & \multicolumn{2}{|c|}{ Meridional Equation } & \multicolumn{2}{|c|}{ Vertical Equation } \\
\hline Term & $\left(\mathrm{m} \mathrm{s}^{-2}\right)$ & Term & $\left(\mathrm{ms}^{-2}\right)$ & Term & $\left(\mathrm{m} \mathrm{s}^{-2}\right)$ \\
\hline$\frac{1}{\rho} \frac{\partial P}{\partial x}$ & $10^{-3}$ & $\frac{1}{\rho} \frac{\partial P}{\partial y}$ & $10^{-3}$ & $\frac{1}{\rho} \frac{\partial P}{\partial z}$ & $10^{1}$ \\
\hline$\frac{u v}{a} \tan \phi$ & $10^{-5}$ & $\frac{u^{2}}{a} \tan \phi$ & $10^{-3}$ & $g$ & $10^{1}$ \\
\hline$\frac{u w}{a}$ & $10^{-5}$ & $\frac{v w}{a}$ & $10^{-6}$ & $\frac{u^{2}+v^{2}}{a}$ & $10^{-3}$ \\
\hline $2 \Omega \cdot v \sin \phi$ & $10^{-6}$ & $2 \Omega \cdot u \sin \phi$ & $10^{-6}$ & $2 \Omega \cdot u \cos \phi$ & $10^{-5}$ \\
\hline $2 \Omega \cdot w \cos \phi$ & $10^{-7}$ & & & & \\
\hline
\end{tabular}
can first obtain the solution for waves under solely horizontal
Table 6

Orders of Magnitude of the Highest Value Found for the Main Terms in the Momentum Equations for the Cloud Region of Venus

Note. These values have been obtained using our updated Venus reference atmosphere.

shear, thus determining the local wave structure at the local wind profile ("local" means here "at a given height"). Once these local solutions are extracted, the wave's overall amplitude is calculated using the wave action equation which directly introduces the vertical variation in the wind (Boyd 1978). Boyd also demonstrated that the knowledge of the meridional shear of the background wind is sufficient to calculate all the wave properties at a given height except for the overall amplitude and phase factors.

The method of multiple scales can be applied if the vertical wave scale $H_{\text {wave }}$ (i.e., one vertical wavelength divided by $2 \pi$ ) is small compared with the vertical scale of the variation for the background zonal wind $\left(H_{\text {wind }}=\left|z_{f}-z_{i}\right|\right)$. The parameter $H_{\text {wind }}$ can be reasonably defined as the vertical distance for which the zonal wind doubles its value $\left(\left|u_{0}\left(z_{f}\right)\right|=2 \cdot\left|u_{0}\left(z_{i}\right)\right|\right)$, and it is displayed for the region of study in panel (A) of Figure 7:

$$
\frac{2 \cdot\left|u_{0}\left(z_{i}\right)\right|-\left|u_{0}\left(z_{i}\right)\right|}{H_{\text {wind }}}=\left|\frac{\partial u_{0}}{\partial z}\right| \Rightarrow H_{\text {wind }}=\left|\frac{u_{0}\left(z_{i}\right)}{\partial u_{0} / \partial z}\right| \text {. }
$$

Unfortunately, measurements of the vertical wavelength for the atmospheric waves on Venus are scarce. Seiff et al. (1992) presented an excellent summary of the waves of Venus, highlighting that vertical wavelengths in the range $700 \mathrm{~m}-1 \mathrm{~km}$ were found during the descent of the Pioneer Venus probes. Later radio-occultation measurements by the Magellan (Hinson \& Jenkins 1995) and Venus Express orbiters (Tellmann et al. 2012) also found wave activity with typical vertical wavelengths less than $8 \mathrm{~km}$ in the former and even smaller (around $3 \mathrm{~km}$ ) in the latter. According to these results, we can define a maximum vertical wave scale of approximately $H_{\text {wave }} \approx 1.3 \mathrm{~km}$ and compare it with the vertical scale of the background zonal wind in order to find the regions where $H_{\text {wave }}$ is small compared to $H_{\text {wind }}$, typically the region where $5 \cdot H_{\text {wave }}<H_{\text {wind }}$. The maximum vertical wavelength which allows one to apply the method of multiple scales on Venus is displayed in panel (B) of Figure 7, where we check that in most of the regions vertical wavelengths higher than $10 \mathrm{~km}$ are allowed.

\section{APPENDIX C}

\section{OPTIMUM AXIS ROTATION}

If we apply the method of perturbations to the momentum equations of a cyclostrophic atmosphere defined in local ENU coordinates, we obtain for the zonal, meridional, and vertical 

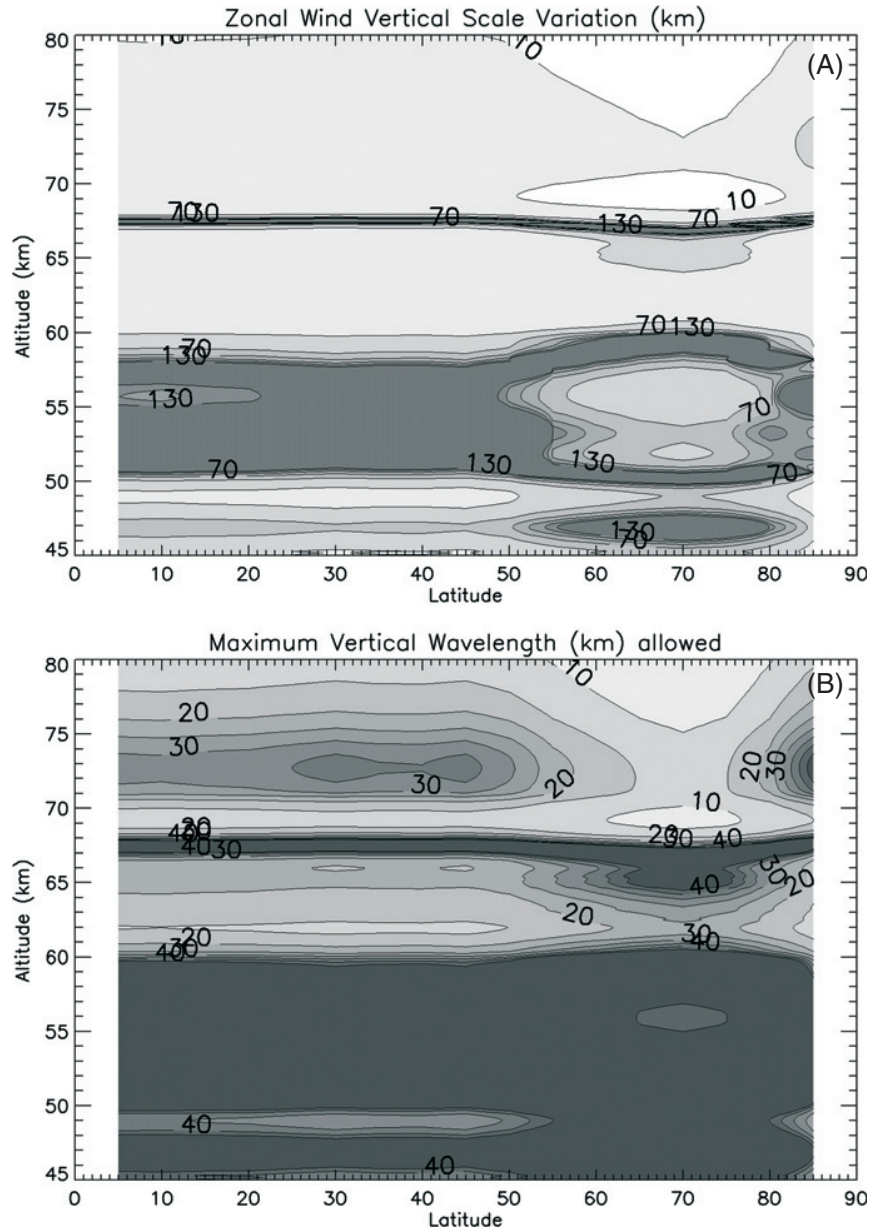

Figure 7. Applicability of the method of the multiple scales on Venus. Panel (A) displays the vertical scale of variation for the zonal wind, while panel (B) exhibits the maximum values of vertical wavelength permitted so that a wave can be described with the dispersion relations deduced in this work.

directions

$$
\begin{gathered}
\frac{\partial u^{\prime}}{\partial t}+u_{0} \frac{\partial u^{\prime}}{\partial x}+\frac{\partial}{\partial x}\left(\frac{P^{\prime}}{\rho_{0}}\right)-\Psi \cdot v^{\prime}+\frac{\partial u_{0}}{\partial y} v^{\prime}=0, \\
\frac{\partial v^{\prime}}{\partial t}+u_{0} \frac{\partial v^{\prime}}{\partial x}+2 \Psi \cdot u^{\prime}+\Psi u_{0} \cdot \frac{\rho^{\prime}}{\rho_{0}}=0 \\
\frac{\partial w^{\prime}}{\partial t}+u_{0} \frac{\partial w^{\prime}}{\partial x}+\frac{1}{\rho_{0}} \frac{\partial P^{\prime}}{\partial z}+\frac{\rho^{\prime}}{\rho_{0}} g=0
\end{gathered}
$$

where the terms $-\Psi \cdot v^{\prime}$ and $2 \Psi \cdot u^{\prime}$ in the zonal and meridional momentum equations (Equations $(\mathrm{Cla})$ and $(\mathrm{C} 1 \mathrm{~b})$ ) represent the change of the metric or curvature terms due to the velocity perturbations projected onto the $x$-axis and $y$-axis, respectively. These metric terms do not alter the gravitational force and are hardly influenced by the rotation of the coordinate system. On the other hand, the terms $\Psi u_{0} \cdot \rho^{\prime} / \rho_{0}$ and $g \cdot \rho^{\prime} / \rho_{0}$ in the meridional and vertical momentum equations represent the influence of the density perturbation on the $y$-component of the centrifugal force associated with the background wind $\left(u_{0}\right)$ and on the gravitational force. Specifically, the term $\Psi u_{0} \cdot \rho^{\prime} / \rho_{0}$ from Equation $(\mathrm{C} 1 \mathrm{~b})$ introduces an undesirable dependence on the density perturbation in the meridional momentum equation that severely complicates the analytical solution of the wave disturbances.
A workaround to this problem is to have the term $\Psi u_{0} \cdot \rho^{\prime} / \rho_{0}$ canceled by $g \cdot \rho^{\prime} / \rho_{0}$ by rotating the coordinate system of the ENU frame around the $x$-axis so that the $z$-axis lies along the vector sum of the gravitational acceleration and the centrifugal force, similar to the convention of standard meteorology where the centrifugal force arises from the rotation of the planet (Holton 2004; Vallis 2006). Thus, by rotating the coordinate system by an angle $\Delta \varphi$, we obtain the following projections of the centrifugal and gravitational forces on the new $y$-axis:

$$
\begin{gathered}
\Re \otimes \boldsymbol{F}_{\mathrm{cf}}=\left(\begin{array}{ccc}
1 & 0 & 0 \\
0 & \cos \Delta \varphi & -\sin \Delta \varphi \\
0 & \sin \Delta \varphi & \cos \Delta \varphi
\end{array}\right) \otimes\left(\begin{array}{c}
-(u v / a) \cdot \tan \phi \\
\left(u^{2} / a\right) \cdot \tan \phi \\
\left(u^{2}+v^{2}\right) / a
\end{array}\right), \\
\Re \otimes g=\left(\begin{array}{ccc}
1 & 0 & 0 \\
0 & \cos \Delta \varphi & -\sin \Delta \varphi \\
0 & \sin \Delta \varphi & \cos \Delta \varphi
\end{array}\right) \otimes\left(\begin{array}{c}
0 \\
0 \\
-g
\end{array}\right), \quad(\mathrm{C} 2 \mathrm{~b})
\end{gathered}
$$

where $\Re$ is the rotation matrix. If we let $\Delta \varphi$ be the angle necessary for the gravitational force to compensate the centrifugal force, we have

$$
-\frac{u^{2}}{a} \tan \phi \cdot \cos \Delta \varphi-\frac{u^{2}+v^{2}}{a} \cdot \sin \Delta \varphi=g \cdot \sin \Delta \varphi .
$$

If we now sort the terms and apply the scale analysis derived for the cyclostrophic atmosphere of Venus in Table 6 (see Appendix A), we obtain

$$
-\frac{u^{2}}{a} \tan \phi \cdot \cos \Delta \varphi=\left(g+\frac{u^{2}+v^{2}}{a}\right) \cdot \sin \Delta \varphi \cong g \cdot \sin \Delta \varphi,
$$

and, dividing by $\cos \Delta \varphi$, it is straightforward to obtain the angle for the optimum frame rotation:

$$
\tan \Delta \varphi=\frac{u_{0}^{2} \tan \phi}{a \cdot g} .
$$

Considering the order of magnitude for the parameters involved in the equation (see Table 6 in Appendix A) this angle is $|\Delta \varphi| \cong 6 \times 10^{-3}$ degrees. Once the system of coordinates has been rotated, the new meridional and vertical momentum equations will contain terms multiplied by $\sin \Delta \varphi \cong 10^{-4}$ and $\cos \Delta \varphi \cong 1$, and it is simple to show that the equations for the wave disturbances become

$$
\begin{gathered}
\frac{\partial u^{\prime}}{\partial t}+u_{0} \frac{\partial u^{\prime}}{\partial x}+\frac{\partial}{\partial x}\left(\frac{P^{\prime}}{\rho_{0}}\right)-\Psi \cdot v^{\prime}+\frac{\partial u_{0}}{\partial y} v^{\prime}=0, \\
\frac{\partial v^{\prime}}{\partial t}+u_{0} \frac{\partial v^{\prime}}{\partial x}+2 \Psi \cdot u^{\prime}=0 \\
\frac{\partial w^{\prime}}{\partial t}+u_{0} \frac{\partial w^{\prime}}{\partial x}+\frac{1}{\rho_{0}} \frac{\partial P^{\prime}}{\partial z}+\frac{\rho^{\prime}}{\rho_{0}} g=0 .
\end{gathered}
$$

\section{APPENDIX D}

\section{THE VERTICAL MOMENTUM EQUATION IN TERMS OF WAVE DISTURBANCES}

The vertical momentum and ideal gas equations for a cyclostrophic atmosphere are

$$
\frac{\partial w}{\partial t}+u \frac{\partial w}{\partial x}+v \frac{\partial w}{\partial y}+w \frac{\partial w}{\partial z}=-\frac{1}{\rho} \frac{\partial P}{\partial z}-g,
$$




$$
P=\rho R T \text {. }
$$

Applying the theory of perturbations to linearize these equations and neglecting products of perturbations, we get

$$
\begin{gathered}
\frac{\partial w^{\prime}}{\partial t}+u_{0} \frac{\partial w^{\prime}}{\partial x}+\frac{1}{\rho_{0}} \frac{\partial P^{\prime}}{\partial z}+\frac{\rho^{\prime}}{\rho_{0}} g=0, \\
\frac{P^{\prime}}{P_{0}}=\frac{\rho^{\prime}}{\rho_{0}}+\frac{T^{\prime}}{T_{0}},
\end{gathered}
$$

where we have considered that the undisturbed atmosphere is in hydrostatic balance, i.e., $\partial P_{0} / \partial z+\rho_{0} g=0$. Taking into account that

$$
\frac{\partial}{\partial z}\left(\frac{P^{\prime}}{\rho_{0}}\right)=\frac{1}{\rho_{0}} \frac{\partial P^{\prime}}{\partial z}-\frac{P^{\prime}}{\rho_{0}} \frac{\partial \ln \rho_{0}}{\partial z},
$$

it follows that

$$
\begin{gathered}
\frac{\partial w^{\prime}}{\partial t}+u_{0} \frac{\partial w^{\prime}}{\partial x}+\frac{\partial}{\partial z}\left(\frac{P^{\prime}}{\rho_{0}}\right)+\frac{P^{\prime}}{\rho_{0}} \frac{\partial \ln \rho_{0}}{\partial z}+\frac{\rho^{\prime}}{\rho_{0}} g=0 \\
\frac{P^{\prime}}{P_{0}}=\frac{\rho^{\prime}}{\rho_{0}}+\frac{T^{\prime}}{T_{0}} .
\end{gathered}
$$

Now, manipulating Poisson's equation for the basic state, we can obtain the following:

$$
\begin{aligned}
\theta_{0} & =\frac{P_{0}}{\rho_{0} R}\left(\frac{P_{r e f}}{P_{0}}\right)^{R / C_{P}} \Rightarrow \\
\ln \theta_{0} & =\ln \frac{P_{r e f}^{R / C_{P}}}{R}+\left(1-\frac{R}{C_{P}}\right) \ln P_{0}-\ln \rho_{0} \Rightarrow \\
\frac{\partial \ln \theta_{0}}{\partial z} & =\left(1-\frac{R}{C_{P}}\right) \frac{\partial \ln P_{0}}{\partial z}-\frac{\partial \ln \rho_{0}}{\partial z} .
\end{aligned}
$$

Replacing the term $\partial \ln \rho_{0} / \partial z$ in Equation (D4a) and considering that the atmospheric stability is $B \equiv \partial \ln \theta / \partial z$,

$$
\begin{gathered}
\frac{\partial w^{\prime}}{\partial t}+u_{0} \frac{\partial w^{\prime}}{\partial x}+\frac{\partial}{\partial z}\left(\frac{P^{\prime}}{\rho_{0}}\right)-B \frac{P^{\prime}}{\rho_{0}} \\
+\frac{P^{\prime}}{\rho_{0}}\left(1-\frac{R}{C_{P}}\right) \frac{\partial \ln P_{0}}{\partial z}+\frac{\rho^{\prime}}{\rho_{0}} g=0, \\
\frac{P^{\prime}}{P_{0}}=\frac{\rho^{\prime}}{\rho_{0}}+\frac{T^{\prime}}{T_{0}} .
\end{gathered}
$$

Using the hydrostatic balance again,

$$
\begin{gathered}
\frac{\partial w^{\prime}}{\partial t}+u_{0} \frac{\partial w^{\prime}}{\partial x}+\frac{\partial}{\partial z}\left(\frac{P^{\prime}}{\rho_{0}}\right)-B \frac{P^{\prime}}{\rho_{0}} \\
-\frac{P^{\prime}}{P_{0}}\left(1-\frac{R}{C_{P}}\right) \cdot g+\frac{\rho^{\prime}}{\rho_{0}} g=0 \\
\frac{P^{\prime}}{P_{0}}=\frac{\rho^{\prime}}{\rho_{0}}+\frac{T^{\prime}}{T_{0}} .
\end{gathered}
$$

Joining all the terms multiplied by the acceleration of gravity in Equation (D7a) and using Equation (D7b), we arrive at the following expression for the vertical momentum equation:

$$
\frac{\partial w^{\prime}}{\partial t}+u_{0} \frac{\partial w^{\prime}}{\partial x}+\frac{\partial}{\partial z}\left(\frac{P^{\prime}}{\rho_{0}}\right)-B \frac{P^{\prime}}{\rho_{0}}-g\left(\frac{T^{\prime}}{T_{0}}-\frac{R}{C_{P}} \frac{P^{\prime}}{P_{0}}\right)=0 .
$$

Then, dividing Poisson's equations for disturbed and undisturbed states and operating,

$$
\begin{aligned}
\frac{\theta_{0}+\theta^{\prime}}{\theta_{0}} & =\frac{\left(T_{0}+T^{\prime}\right) \cdot\left[P_{\text {ref }} /\left(P_{0}+P^{\prime}\right)\right]^{R / C_{P}}}{T_{0} \cdot\left(P_{\text {ref }} / P_{0}\right)^{R / C_{P}}} \Rightarrow \\
1+\frac{\theta^{\prime}}{\theta_{0}} & =\left(1+\frac{T^{\prime}}{T_{0}}\right) \cdot\left(1+\frac{P^{\prime}}{P_{0}}\right)^{-R / C_{P}} \Rightarrow \\
\ln \left(1+\frac{\theta^{\prime}}{\theta_{0}}\right) & =\ln \left(1+\frac{T^{\prime}}{T_{0}}\right)-\frac{R}{C_{P}} \ln \left(1+\frac{P^{\prime}}{P_{0}}\right) .
\end{aligned}
$$

Taking into account that $\ln (1+x) \cong x$ when $x \ll 1$, we can modify Equation (D8) and get

$$
\frac{\partial w^{\prime}}{\partial t}+u_{0} \frac{\partial w^{\prime}}{\partial x}+\frac{\partial}{\partial z}\left(\frac{P^{\prime}}{\rho_{0}}\right)-B \frac{P^{\prime}}{\rho_{0}}-g \frac{\theta^{\prime}}{\theta_{0}}=0 .
$$

Finally, defining $\Theta \equiv \ln \theta$, we can demonstrate that $\Theta \prime \cong \theta^{\prime} / \theta_{0}$ by again applying $\ln (1+x) \cong x$ when $x \ll 1$ :

$$
\Theta_{0}+\Theta^{\prime}=\ln \left(\theta_{0}+\theta^{\prime}\right)=\ln \theta_{0}+\ln \left(1+\frac{\theta^{\prime}}{\theta_{0}}\right) \cong \ln \theta_{0}+\frac{\theta^{\prime}}{\theta_{0}} .
$$

We finally obtain for the vertical momentum equation

$$
\frac{\partial w^{\prime}}{\partial t}+u_{0} \frac{\partial w^{\prime}}{\partial x}+\frac{\partial}{\partial z}\left(\frac{P^{\prime}}{\rho_{0}}\right)-B \frac{P^{\prime}}{\rho_{0}}-g \cdot \Theta^{\prime}=0 .
$$

\section{APPENDIX E}

\section{TRACER PARAMETERS IN SCALED EQUATIONS}

We demonstrated that the perturbed vertical momentum and continuity equations had the form

$$
\begin{aligned}
& n_{4} \cdot\left(\frac{\partial w^{\prime}}{\partial t}+u_{0} \frac{\partial w^{\prime}}{\partial x}\right)+\frac{\partial}{\partial z}\left(\frac{P^{\prime}}{\rho_{0}}\right)-n_{3} \cdot B \frac{P^{\prime}}{\rho_{0}}-g \cdot \Theta^{\prime}=0, \quad(\mathrm{E} 1 \mathrm{a}) \\
& n_{2} \cdot\left[\frac{\partial}{\partial t}\left(\frac{\rho^{\prime}}{\rho_{0}}\right)+u_{0} \frac{\partial}{\partial x}\left(\frac{\rho^{\prime}}{\rho_{0}}\right)\right]+\frac{\partial u^{\prime}}{\partial x}+\frac{\partial w^{\prime}}{\partial z}-n_{1} \cdot \frac{w^{\prime}}{H_{0}}=0,
\end{aligned}
$$

where $\left(u^{\prime}, w^{\prime}, \rho^{\prime}, P^{\prime}, \Theta^{\prime} \cong \theta^{\prime} / \theta_{0}\right)$ are the wave perturbations for the zonal and vertical wind velocity, atmospheric density, pressure, and potential temperature. On the other hand, $\rho_{0}(z)$ and $u_{0}\left(y, z_{0}\right)$ are the atmospheric density and zonal wind in their basic states, $H_{0}$ is the density scale height, $g$ is the acceleration of gravity, and $B$ is the atmospheric static stability $(B \equiv \partial \ln \theta / \partial z)$ with $N=\sqrt{g B}$ being the Brunt-Väisälä frequency. Finally, $\left(n_{1}, n_{2}, n_{3}, n_{4}\right)$ are the tracer parameters multiplying those terms in the wave disturbance equations that are related to the most relevant assumptions to filter waves (see Section 5): the hydrostatic approximation $(D w / D t=0)$ is applied when setting $n_{4}=0$, an incompressible atmosphere $(D \rho / D t=0)$ is applied when $n_{1}=n_{2}=n_{3}=0$, the atmosphere behaves as intrinsically anelastic (i.e., anelastic relative to the background zonal wind) if we set $n_{2}=n_{3}=0$, while applying $n_{1}=n_{2}=n_{3}=n_{4}=0$ can be demonstrated to be equivalent to applying the Boussinesq approximation.

These tracer parameters can be shown to be linked to certain dimensionless variables related to the atmospheric approximations they involve. We can non-dimensionalize the physical variables (Vallis 2006) involved in Equations (E1):

$$
\left(u^{\prime}, v^{\prime}\right)=U \cdot\left(\tilde{u}^{\prime}, \tilde{v}^{\prime}\right),
$$




$$
\begin{gathered}
w^{\prime}=W \cdot \tilde{w}^{\prime}=\frac{H \cdot U}{L} \cdot \tilde{w}^{\prime}=\alpha \cdot U \cdot \tilde{w}^{\prime}, \\
(x, y, z)=(\tilde{x} \cdot L, \tilde{y} \cdot L, \tilde{z} \cdot H), \\
t=\tilde{t} \cdot \tau=\tilde{t} \cdot \frac{L}{U}, \\
P^{\prime}=\tilde{P}^{\prime} \cdot \rho_{0} \cdot U^{2},
\end{gathered}
$$

where $\tau, U, L, W, H$ are the timescale, horizontal velocity and length scales, and vertical velocity and length scales, respectively, and $\alpha$ is the aspect ratio parameter. Introducing the expressions (E2) into the perturbed equations (Equation (E1)), we obtain

$$
\begin{aligned}
n_{4} \cdot \alpha \cdot \frac{U^{2}}{L} \cdot\left(\frac{\partial \tilde{w}^{\prime}}{\partial \tilde{t}}+\tilde{u}_{0} \frac{\partial \tilde{w}^{\prime}}{\partial \tilde{x}}\right)+\frac{U^{2}}{H} \cdot \frac{\partial \tilde{P}^{\prime}}{\partial \tilde{z}} \\
-n_{3} \cdot U^{2} \frac{N^{2}}{g} \tilde{P}^{\prime}-g \cdot \Theta^{\prime}=0, \\
n_{2} \cdot \frac{U}{L} \cdot\left[\frac{\partial}{\partial \tilde{t}}\left(\frac{\rho^{\prime}}{\rho_{0}}\right)+\tilde{u}_{0} \frac{\partial}{\partial \tilde{x}}\left(\frac{\rho^{\prime}}{\rho_{0}}\right)\right]+\frac{U}{L} \cdot \frac{\partial \tilde{u}^{\prime}}{\partial \tilde{x}} \\
+\alpha \cdot \frac{U}{H} \cdot \frac{\partial \tilde{w}^{\prime}}{\partial \tilde{z}}-n_{1} \cdot \alpha \cdot U \cdot \frac{\tilde{w}^{\prime}}{H_{0}}=0 .
\end{aligned}
$$

Several dimensionless parameters can be introduced now: the Mach number $\left(M^{2} \approx \rho^{\prime} / \rho_{0}\right)$, which defines the degree of compressibility of the fluid, the Richardson number $\left(\mathrm{Ri} \equiv g H / U^{2}\right)$, which expresses the ratio of potential to kinetic energy, and the vertical Froude number $\left(F r_{V} \equiv U /[H \cdot N]\right)$, which provides a measure of the stratification of the flow. Operating on Equations (E3) we finally have the vertical momentum and continuity equations without dimensions:

$$
\begin{gathered}
n_{4} \operatorname{Fr}_{V} \alpha \cdot\left(\frac{\partial \tilde{w}^{\prime}}{\partial \tilde{t}}+\tilde{u}_{0} \frac{\partial \tilde{w}^{\prime}}{\partial \tilde{x}}\right)+\frac{\operatorname{Fr}_{V}}{\alpha} \cdot \frac{\partial \tilde{P}^{\prime}}{\partial \tilde{z}} \\
-\frac{M^{2}}{\alpha} \cdot\left(\frac{n_{3}}{\operatorname{Fr}_{V} \cdot \operatorname{Ri}} \cdot \tilde{P}^{\prime}+\operatorname{Fr}_{V} \cdot \operatorname{Ri} \cdot \Theta^{\prime}\right)=0, \\
n_{2} \cdot M^{2} \cdot\left[\frac{\partial}{\partial \tilde{t}}\left(\frac{\rho^{\prime}}{\rho_{0}}\right)+\tilde{u}_{0} \frac{\partial}{\partial \tilde{x}}\left(\frac{\rho^{\prime}}{\rho_{0}}\right)\right]+\frac{\partial \tilde{u}^{\prime}}{\partial \tilde{x}}+\frac{\partial \tilde{w}^{\prime}}{\partial \tilde{z}} \\
-n_{1} \cdot M^{2} \cdot \alpha \cdot \frac{L}{H_{0}} \cdot \tilde{w}^{\prime}=0,
\end{gathered}
$$

where the Mach number appears in Equation (E4b) multiplying those terms coming from $D\left(\rho^{\prime} / \rho_{0}\right) / D t$, and in the case of Equation (E4a) coming from a term containing $\rho^{\prime} / \rho_{0}$ (see Equation (3c) and Appendix D). For subsonic fluids $\left(M^{2} \ll 1\right)$, the fluid can be considered as incompressible and setting a small value for the Mach number is, thus, equivalent to setting $n_{1}=$ $n_{2}=n_{3}=0$, precisely the tracer parameters multiplying the Mach number. The hydrostatic balance is associated with a small value of the aspect ratio parameter $\left(\alpha^{2} \ll 1\right)$ and, in the more generic case of a stratified fluid, to small values of the product between the Froude number and the aspect ratio $\left(\alpha^{2} \cdot \operatorname{Fr}_{V}^{2} \ll 1\right)$ (Vallis 2006). This is clearly linked to setting $n_{4}=0$ in the vertical momentum equation (Equation (E4a)) in order to apply the hydrostatic balance. Concerning the Richardson number, when this is much lower than unity, buoyancy is negligible in the flow, while when being of the order of unity or higher, the flow is likely to be buoyancy-driven. Observe that, consistently, the Richardson number appears in the vertical momentum equation (Equation (E4a)), multiplying the perturbations on the atmospheric potential temperature.

\section{REFERENCES}

Apt, J., \& Leung, J. 1982, Icar, 49, 427

Belton, M. J. S., Smith, G. R., Schubert, G., \& Del Genio, A. D. 1976, JAtS, 33, 1394

Bird, M. K., Allison, M., \& Asmarm, S. W. 2005, Natur, 438, 800

Boyd, J. P. 1978, JAtS, 35, 2236

Covey, C. C., \& Schubert, G. 1981, Icar, 47, 130

Covey, C. C., \& Schubert, G. 1982, JAtS, 39, 2397

Del Genio, A. D., \& Rossow, W. B. 1990, JAtS, 47, 293

de Pater, I., \& Lissauer, J. J. 2001, Planetary Sciences (Cambridge: Cambridge Univ. Press)

Durran, D. R. 2008, JFM, 601, 365

Eckart, C. 1960, Hydrodynamics of Oceans and Atmospheres (Oxford: Pergamon)

Faigler, S., Tal-Or, L., Mazeh, T., Latham, D. W., \& Buchhave, L. A. 2013, ApJ, 771,26

Flasar, F. M., Baines, K. H., Bird, M. K., Tokano, T., \& West, R. A. 2010, in Titan from Cassini-Huygens, ed. R. H. Brown, J.-P. Lebreton, \& J. H. Waite (Berlin: Springer), 323

Gierasch, P. J., Goody, R. M., Young, R. E., et al. 1997, in Venus II: Geology, Geophysics, Atmosphere, and Solar Wind Environment, ed. S. W. Bougher, D. M. Hunten, \& R. J. Philips (Tucson, AZ: Univ. Arizona Press), 459

Gill, A. E. 1982, in Atmosphere-Ocean Dynamics, ed. L. D. William (London: Academic Press), 237

Green, J. 1999, Atmospheric Dynamics (Cambridge Atmospheric and Space Science Series; Cambridge: Cambridge Univ. Press)

Heng, K., Menou, K., \& Phillipps, P. J. 2011, MNRAS, 413, 2380

Hinson, D. P., \& Jenkins, J. M. 1995, Icar, 114, 310

Holton, J. R. 2004, An Introduction to Dynamic Meteorology (4th ed.; New York: Elsevier)

Holton, J. R., Pyle, J., \& Curry, J. A. 2002, Encyclopedia of Atmospheric Sciences (Amsterdam: Elsevier)

Hou, A. Y., \& Farrell, B. F. 1987, JAtS, 44, 1049

Hueso, R., Peralta, J., \& Sánchez-Lavega, A. 2012, Icar, 217, 585

Iga, S. I., \& Matsuda, Y. 2005, JAtS, 62, 2514

Imamura, T. 2006, JAtS, 63, 1623

Joshi, M. M., Haberle, R. M., \& Reynolds, R. T. 1997, Icar, 129, 450

Kasting, J. F., Whitmire, D. P., \& Reynolds, R. T. 1993, Icar, 101, 108

Knutson, H. A., Charbonneau, D., Allen, L. E., et al. 2007, Natur, 447, 183

Knutson, H. A., Charbonneau, D., Cowan, N. B., et al. 2009, ApJ, 690, 822

Lebonnois, S., Hourdin, F., Eymet, V., et al. 2010, JGRE, 115, E06006

Lee, C., Lewis, S. R., \& Read, P. L. 2007, JGRE, 112, E04S11

Leroy, S. S., \& Ingersoll, A. P. 1995, JAtS, 52, 3717

Lorenz, R. D., Young, L. A., \& Ferri, F. 2014, Icar, 227, 49

Luz, D., Berry, D. L., Piccioni, G., et al. 2011, Sci, 332, 577

Luz, D., Civeit, T., Courtin, R., et al. 2006, JGRE, 111, E08S90

Machado, P., Luz, D., Widemann, T., Lellouch, E., \& Witasse, O. 2012, Icar, 221,248

Merlis, T. M., \& Schneider, T. 2010, JAMES, 2, 13

Nicholls, M. E., \& Pielke, R. A. 2000, JAtS, 57, 3251

Norbury, J., \& Roulstone, I. 2002, Large-Scale Atmosphere-Ocean Dynamics (Cambridge: Cambridge Univ. Press)

Peale, S. J. 1977, in Planetary Satellites, ed. J. A. Burns (Tucson, AZ: Univ. Arizona Press)

Peralta, J., Hueso, R., \& Sánchez-Lavega, A. 2007, Icar, 190, 469

Peralta, J., Hueso, R., Sánchez-Lavega, A., et al. 2008, JGRE, 113, E00B18

Peralta, J., Imamura, T., Read, P. L., Luz, D., \& Piccialli, A. 2014, ApJS, 213, 18

Peralta, J., Luz, D., Berry, D. L., et al. 2012, Icar, 220, 958

Petculescu, A., \& Lueptow, R. M. 2007, Icar, 186, 413

Piccialli, A. 2010, PhD thesis, Technische Univ. Braunschweig

Piccialli, A., Titov, D. V., Sánchez-Lavega, A., et al. 2014, Icar, 227, 94

Rauscher, E., \& Menou, K. 2012, ApJ, 750, 96

Read, P. L. 2011, P\&SS, 59, 900

Rossow, W. B., Del Genio, A., Limaye, S. S., \& Travis, L. D. 1980, JGR, 35,8107

Rossow, W. B., Del Genio, A. D., \& Eichler, T. 1990, JAtS, 47, 2053

Schinder, P. J., Gierasch, P. J., Leroy, S. S., \& Smith, M. D. 1990, JAtS, 47, 2037

Schneider, J., Dedieu, C., Le Sidaner, P., Savalle, R., \& Zolotukhin, I. 2011, A\&A, 532, A79

Schubert, G. 1983, in Venus, ed. D. Hunten, L. Colin, T. Donahue, \& V. Moroz (Tucson, AZ: Univ. Arizona Press), 681 
Schubert, G., \& Walterscheid, R. L. 1984, JAtS, 41, 1202

Seiff, A., Schofield, J. T., Kliore, A. J., et al. 1985, AdSpR, 5, 3

Seiff, A., Young, R. E., Haberle, R., \& Houben, H. 1992, in Proceedings of the Chapman Conference, Venus and Mars: Atmospheres, Ionospheres, and Solar Wind Interactions, ed. J. G. Luhmann, M. Tatrallyay, \& R. O. Pepin (Washington, DC: American Geophysical Union), 73

Showman, A. P., Fortney, J. J., Lian, Y., et al. 2009, ApJ, 699, 564

Showman, A. P., \& Polvani, L. M. 2011, ApJ, 738, 71

Smith, M. D., Gierasch, P. J., \& Schinder, P. J. 1992, Sci, 256, 652

Smith, M. D., Gierasch, P. J., \& Schinder, P. J. 1993, JAtS, 50,4080
Snellen, I. A. G., de Kok, R. J., de Mooij, E. J. W., \& Albrecht, S. 2010, Natur, 465, 1049

Taylor, F. W., Beer, R., Chahine, M. T., et al. 1980, JGR, 85, 7963

Tellmann, S., Häusler, B., Hinson, D. P., et al. 2012, Icar, 221, 471

Tellmann, S., Pätzold, M., Häusler, B., Bird, M. K., \& Tyler, G. L. 2009, JGRE, 114, E00B36

Vallis, G. K. 2006, Atmospheric and Oceanic Fluid Dynamics (Cambridge: Cambridge Univ. Press)

Yamamoto, M., \& Takahashi, M. 2003, JAtS, 60, 561

Zaitsev, V. F., \& Polyanin, A. D. 2002, Handbook of Exact Solutions for Ordinary Differential Equations (2nd ed.; Boca Raton, FL: CRC Press), 816 